\title{
A semivectorial bilevel programming approach to optimize electricity dynamic time-of-use retail pricing
}

\author{
Maria João Alves 1,3,*, Carlos Henggeler Antunes ${ }^{2,3}$ \\ 1 CeBER and Faculty of Economics, University of Coimbra, Av. Dias da Silva 165, 3000 Coimbra, \\ Portugal
}

2 Dept. of Electrical and Computer Engineering, University of Coimbra, Polo 2, 3030-290 Coimbra, Portugal

3 INESC Coimbra, Polo 2 - DEEC, 3030-290 Coimbra, Portugal

mjalves@fe.uc.pt; ch@deec.uc.pt;

\begin{abstract}
Presently, residential electricity consumers are, in general, charged at flat or dual time-of-use tariffs along the day, which are defined by the retailer for long periods (e.g., one year). These pricing schemes do not convey price signals reflecting generation costs and grid conditions. Hence, consumers lack the incentives to engage in different consumption patterns using the flexibility they generally have in the operation of some end-use loads. Dynamic tariffs, i.e. energy prices varying possibly with significant magnitude in short periods of time, are expected to become an applicable pricing scheme in smart grids. In this setting, home energy management systems can play an important role to help end-users optimizing the usage of appliances to minimize energy costs without compromising comfort. This can be advantageous also from the perspective of grid management.
\end{abstract}

A semivectorial bilevel programming approach is developed to model the interaction between electricity retailers and consumers in order to optimize electricity time-of-use retail pricing. The aim is to support the retailer in finding good decisions for the prices. The retailer (upper level decision maker) establishes dynamic time-of-use electricity prices to maximize profits. The consumer (lower level decision maker) responds by selecting, under that price setting, a load scheduling decision leading to a nondominated solution balancing his objectives of minimizing the electricity bill (cost dimension) and minimizing the dissatisfaction in face of his preferences and requirements (comfort dimension).

The lower level optimization problem is formulated as a bi-objective mixed-integer linear programming problem. A hybrid approach is proposed, which consists of a genetic algorithm for the upper level problem and an exact solver to solve surrogate scalar problems at the lower level. A case study is presented and discussed.

Keywords: Semivectorial bilevel optimization; Multi-objective optimization; Genetic algorithms; Hybrid approaches; Demand response; Electricity retail markets.

\section{Introduction}

In several countries, the electricity sector has been open to retail competition, including to residential customers. Under deregulation, retailers procure electricity in wholesale markets in which prices are generally established by locational marginal pricing schemes in day-ahead markets; retailers then offer flat or (slightly) variable time-of-use tariffs to their residential customers, thus managing the risk involved. The price signal is conveyed to consumers in flat or more usual dual time-of-use tariffs (i.e., considering two differently priced periods within one day such as peak and off-peak), which are defined for long periods (e.g., one year). Therefore, this type of price signal does not reflect varying generation costs and grid conditions. Moreover, negative wholesale prices have occurred sporadically in periods of

* Corresponding author 
excess of generation based on renewable sources and low demand [1][2]. In this context, the setting of time-differentiated tariffs at the retail level reflecting the power systems conditions (generation availability, network congestion, etc.) has the potential to contribute to improving the overall efficiency of the system, lowering peak generation costs, facilitating the penetration of renewable sources, reducing network losses and contributing to the deferral of network reinforcement investments, while offering economic benefits to customers. The implementation of these dynamic tariffs, i.e. energy prices varying possibly with significant magnitude in short periods of time, will be facilitated by the deployment of smart meters, underpinning the empowerment of consumers in the evolution of electricity network to smart grids. These price incentives will motivate consumers to engage in different consumption patterns, namely by using the flexibility they generally have in the operation of some appliances (end-use loads) through adequate demand response actions. After receiving tariff information some time in advance (e.g. one day), the consumer is able to respond by scheduling load operation providing energy services, such as hot water, laundry, electric vehicle charging, etc. Different schedules represent distinct trade-offs between the minimization of the electricity bill and the minimization of the dissatisfaction associated with postponing or anticipating load operation to different periods. The optimization of these cost and comfort dimensions will be made operational by a home energy management system, such as the one proposed in [3]. This system can be parameterized with the customer's energy usage profile and is endowed with communication capabilities to receive grid information (prices and possibly emergency requests) and switch the loads through on/off plugs. These smart technologies are recognized by the European Parliamentary Research Service [4] "as a way to encourage greater consumer involvement in the retail market, facilitate dynamic pricing and foster demand response", linking "smart metering systems with smart appliances that can automatically adjust their energy use according to price signals".

In the present work, we formulate the problem of optimizing electricity time-of-use retail pricing as a semivectorial bilevel optimization model, i.e., a bilevel optimization model with a single objective function at the upper level and multiple objective functions at the lower level. Bilevel optimization models and game-theoretical approaches have been proposed to determine equilibrium solutions in problems related with the interaction between electricity retailers and consumers. In a bilevel decision problem, the decision maker at the upper level is the leader and the decision maker at the lower level is the follower. The leader decides first, but his objective is affected by the follower's decision.

Zhang et al. [5] proposed a multi-leader/single-follower nonlinear bilevel optimization model to analyze the strategic bidding behavior of generating companies (leaders) in day-ahead electricity markets. This model assists generators in choosing their biddings to maximize profits and a market operator (follower) to minimize its purchase electricity costs. The multiple leaders and the follower have individual control variables, objectives and constraints. The model is tackled using a particle swarm optimization approach.

Meng and Zeng [6] proposed a bilevel optimization model in which the electricity retailer (leader) determines real-time retail prices to maximize profit and the customer (follower) reacts to minimize electricity bills. The hierarchical problem is converted into one single level problem by replacing the lower level problem with the Karush-Kuhn-Tucker (KKT) conditions. A branch and bound algorithm is then used to solve the resulting single level problem as in [7]. Meng and Zeng [8] proposed a real-time pricing scheme for demand response management in smart grids by means of a single-leader/multifollower model, in which the retailer determines the price and the customers' energy management systems automatically manage the energy usage by appliances in the households to maximize benefits. In the lower level, three separate linear sub-problems corresponding to interruptible, non-interruptible and curtailable appliances are considered. Meng and Zeng [9] extended the previous model to include also costumers whose energy consumption patterns are not known by the retailer. Therefore, the retailer should learn these patterns with the purpose of determining the retail prices. Meng and Zeng [10] further introduced a waiting time cost model for modeling the consumer's energy cost of interruptible and noninterruptible appliances. In this model, a waiting time benefit function is defined to represent the energy bill saved by waiting a given time before the operation of appliances. The approaches [8], [9] and [10] use genetic algorithms to maximize the retailer's profit, determining the optimal real-time pricing scheme considering the expected customers' reactions. An LP solver is used to optimize separately each subproblem at the lower level.

Zugno et al. [11] proposed a model to determine the dynamic price-signal delivering maximum retailer profit and the corresponding optimal load pattern for consumers, subject to stochastic prices, weather- 
related variables and must-serve load. The bilevel model is reformulated as a single level mixed-integer linear programming (MILP) problem.

Alves et al. [12] proposed a hybrid approach consisting of a genetic algorithm and an exact MILP solver to deal with a bilevel problem in which the retailer establishes prices to maximize profits and consumers are able to deviate consumption of shiftable loads to minimize costs. Constraints impose specific time slots for load operation, which may decrease the retailer's profit. Carrasqueira et al. [13] proposed two bilevel population-based algorithms, one based on an evolutionary algorithm and the other on particle swarm optimization, to tackle the same problem.

Sekizaki et al. [14] presented a bilevel electricity retail market model in which flexible responses of consumers are traded at selling prices offered by a retailer over one day, considering distribution network physical constraints. A genetic algorithm is used to find an approximated solution to the non-convex bilevel model.

$\mathrm{Bu}$ et al. [15] developed a game-theoretical decision-making scheme for electricity retailers in the smart grid, using real-time pricing demand-side schemes. Various utility functions to model the electricity customers' preferences and electricity consumption patterns are used. Customers adjust electricity demand to maximize their individual utility, which depends on the amount of energy consumed, the price and an individual factor. The retailer aims at maximizing profit. Simulations have been carried out to study how the system parameters affect the decisions. Yang et al. [16] also developed a game-theoretical approach to optimize time-of-use pricing strategies, which includes models of costs to utility companies arising from demand fluctuations and models of user satisfaction. The user satisfaction depends on the difference between the nominal demand and the actual consumption. Utility functions are designed for both players, considering scenarios with a single user type and multiple user types responding differently to time-varying prices.

Saez-Gallego et al. [17] aimed to capture the demand response of a pool of flexible (price-responsive) consumers determining the corresponding optimal market bid. That is, electricity prices are exogenous and not control variables. An inverse optimization framework using a time series of price-consumption pairs is developed resulting in a bilevel optimization problem. The inverse problem aims at estimating the parameters defining the objective function and the constraints of the lower level problem in order to minimize the difference between the optimal consumption and the measured consumption.

The bilevel models mentioned above do not include the operation cycles of the household appliances (apart from the model proposed in [12]). The operation cycles define the power requested to the grid by each appliance in each stage of operation (e.g. water heating, centrifugation, etc., in washing machine operation) and their incorporation into the model is essential to obtain a realistic load characterization. In addition, the representation of the consumer's objectives in these studies has been reduced to the minimization of the electricity bill or some type of (difficult to elicit) utility functions of consumer's satisfaction. In the present paper, we consider explicitly two objective functions at the consumer's level representing cost and comfort dimensions. Consumer's decisions are modeled at appliance level, considering typical operation cycles of several appliances and information about the time slots preferred for operation.

A semivectorial bilevel optimization model is proposed to assist the retailer in optimizing dynamic electricity tariffs varying in sub-periods during one day (upper level problem). This model is based on the scalar bilevel model presented in [12]. The retailer is the leader and wishes to determine tariffs that maximize his profit. The consumer is the follower who reacts to make the most of these timedifferentiated prices to minimize his electricity bill (cost dimension) and minimize the dissatisfaction (comfort dimension) associated with rescheduling load operation according to the flexibility resulting from his preferences and requirements. The model considers a cluster of consumers with similar consumption and demand response profiles. The lower level optimization problem is formulated as a multi-objective mixed-integer linear programming (MOMILP) model. The consumer's decision consists of determining a load schedule corresponding to a nondominated solution trading-off his two objective functions.

The proposed model is tackled using a hybrid approach consisting of a genetic algorithm for the upper level problem and an exact solver to solve surrogate scalar problems (i.e. combining both objective functions) at the lower level. The consideration of multiple objective functions at the lower level 
introduces further challenges regarding computational difficulties to ensure that solutions are actually nondominated and the need for the retailer to examine a set of extreme outcomes resulting from his optimistic/pessimistic stance and the possible consumer's reaction. In this setting, the computation of four extreme (optimistic, pessimistic, deceiving and rewarding) solutions offers comprehensive information for the retailer's decision process. The aim is to support the retailer in finding good decisions for the prices, although these may not be the optimal extreme solutions to the model due to the use of the genetic algorithm for the upper level problem. No other approaches have been found in the literature to deal with a model with these characteristics.

The structure of the paper is as follows. In section 2, semivectorial bilevel programming is introduced. Four types of extreme solutions (the optimistic, deceiving, pessimistic and rewarding solutions) are introduced, which provide useful insights for the best outcome of the decision process under different perspectives of analysis. In section 3, the semivectorial bilevel model with a MOMILP problem (with two objective functions) at the lower level is presented to study the interactions between the electricity retailer and consumers. The semivectorial hybrid genetic algorithm developed to compute the four extreme solutions is presented in section 4. An illustrative case study based on real data is presented and discussed in section 5, including the incorporation of consumer's preferences to compute nondominated compromise solutions other than the extreme solutions. In section 6, the main conclusions are drawn.

\section{Semivectorial bilevel programming}

A general bilevel programming problem with a single objective function at each level can be defined as follows:

$$
\begin{array}{ll}
\max _{x} " \quad F(x, y) & \\
\text { s.t. } & G(x, y) \leq 0 \\
& y \in \arg \min _{y}\{f(x, y): g(x, y) \leq 0\}
\end{array}
$$

where $x \in \mathfrak{R}^{n_{1}}$ is a $n_{1}$-dimensional vector of upper level variables and $y \in \mathfrak{R}^{n_{2}}$ is a $n_{2}$-dimensional vector of lower level variables; $F(x, y)$ and $f(x, y)$ are the leader's and the follower's objective functions, respectively. Since the follower optimizes $f(x, y)$ after $x$ has been selected, $x$ assumes a constant vector whenever $f(x, y)$ is optimized.

The follower's rational reaction set to a given $x^{\prime}$ is:

$$
\Psi\left(x^{\prime}\right)=\arg \min _{y}\left\{f\left(x^{\prime}, y\right): g\left(x^{\prime}, y\right) \leq 0\right\} .
$$

The feasible region for the bilevel problem (BP) is called the inducible region:

$$
I R=\left\{(x, y) \in \mathfrak{R}^{n_{1}+n_{2}}: G(x, y) \leq 0, y \in \Psi(x)\right\} \text {. }
$$

Without loss of generality, problem (BP) has been defined with a maximizing upper level function and a minimizing lower level function to be closer to the bilevel problem dealing with the interaction between an electricity retailer and consumers addressed in this paper. Nothing substantial changes if both objective functions are to be minimized or maximized.

The bilevel problem is the leader's problem. Quotation marks have been added in "max" $F(x, y)$ due to the unclear definition of the objective function value $F(x, y)$ from the leader's point-of-view (who has control over $x$ only) if the set of optimal solutions of the lower level problem is not singleton [18]. Multiple optimal responses of the follower pose a problem to the leader, as he does not know which optimal solution the follower will choose. This choice may affect significantly the leader's objective value and, consequently, his decision. In many studies this difficulty is avoided just by assuming that the solution to the lower level problem is unique. In other cases, an optimistic or a pessimistic approach is considered. The optimistic approach (the most common one) assumes that the leader is free to select the solution that suits him best among the optimal decisions of the follower. In this case, the upper level optimization is taken with respect to $x$ and $y$, meaning that the leader is able to influence the choice of the follower [18]. When cooperation between the leader and the follower is not allowed, or if the leader is risk-averse and wishes to bound the damage resulting from an undesirable selection of the follower, a 
pessimistic approach may be admitted. The leader prepares for the worst case, that is, he chooses a decision $x$ which performs best in view of the 'worst' (globally optimal) follower's response [19]. The pessimistic bilevel problem is perceived to be very difficult to solve and, typically, the algorithms for the optimistic bilevel problem do not extend to the pessimistic formulation [20].

A semivectorial bilevel problem is a bilevel programming problem with a scalar optimization problem in the upper level and a multiobjective (vector-valued) optimization problem in the lower level. A general semivectorial bilevel problem (SVBP) with $m$ minimizing objective functions at the lower level and a maximizing objective function at the upper level can be formulated as follows:

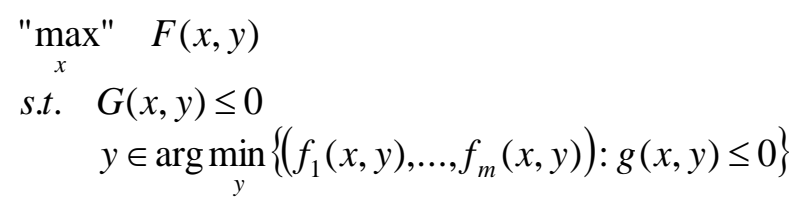

In a semivectorial bilevel problem only efficient (nondominated or Pareto optimal) solutions to the lower level problem for each $x$ vector are feasible to the bilevel problem.

Let $Y(x)=\left\{y \in \mathfrak{R}^{n_{2}}: g(x, y) \leq 0\right\}$.

For a given $x^{\prime}$ :

- a solution $y^{\prime} \in Y\left(x^{\prime}\right)$ is weakly efficient to the lower level problem if and only if there is no other $y \in \mathfrak{R}^{n_{2}}, y \in Y\left(x^{\prime}\right)$, such that $f_{j}\left(x^{\prime}, y\right)<f_{j}\left(x^{\prime}, y^{\prime}\right)$ for all $j=1, \ldots, m$;

- a solution $y^{\prime} \in Y\left(x^{\prime}\right)$ is efficient to the lower level problem if and only if there is no other $y \in \mathfrak{R}^{n_{2}}$, $y \in Y\left(x^{\prime}\right)$, such that $f_{j}\left(x^{\prime}, y\right) \leq f_{j}\left(x^{\prime}, y^{\prime}\right)$ for all $j=1, \ldots, m$ and $f_{j}\left(x^{\prime}, y\right)<f_{j}\left(x^{\prime}, y^{\prime}\right)$ for at least one $j$.

Let $f\left(x^{\prime}, y\right) \prec f\left(x^{\prime}, y^{\prime}\right)$ denote the dominance relation, i.e. $f_{j}\left(x^{\prime}, y\right) \leq f_{j}\left(x^{\prime}, y^{\prime}\right)$ for all $j=1, \ldots, m$ and $f_{j}\left(x^{\prime}, y\right)<f_{j}\left(x^{\prime}, y^{\prime}\right)$ for at least one $j$.

Thus, the set of efficient solutions to the lower level problem of formulation (SVBP) for a given $x^{\prime}$ is defined as: $\Psi_{E f}\left(x^{\prime}\right)=\left\{y^{\prime} \in Y\left(x^{\prime}\right)\right.$ : there is no $y \in Y\left(x^{\prime}\right)$ such that $\left.f\left(x^{\prime}, y\right) \prec f\left(x^{\prime}, y^{\prime}\right)\right\}$.

The feasible region of problem (SVBP) is $I R=\left\{(x, y) \in \mathfrak{R}^{n_{1}+n_{2}}: G(x, y) \leq 0, y \in \Psi_{E f}(x)\right\}$.

The bilevel programming problem with multiple objective functions at the lower level was firstly called semivectorial bilevel optimization problem by Bonnel [21] and Bonnel and Morgan [22]. In [21] necessary optimality conditions for the optimistic formulation were provided and in [22] a penalty function method to tackle this type of problem was proposed. The optimistic formulation assumes that the follower accepts any efficient solution to the lower level problem. Other methods based on penalty functions were further proposed by Ankhili and Mansouri [23], Zheng and Wan [24], Zheng et al. [25] and Ren and Wang [26] for the SVBP with multi-objective linear programming (MOLP) problems in the lower level. Calvete and Galé [27] also focused on bilevel problems with lower level MOLP problems. Assuming a quasiconcave upper level objective function and all constraints linear, an enumerative exact algorithm and a genetic algorithm were proposed based on the search of the extreme points of the polyhedron defined by all constraints. Lv and Wan [28] proposed an algorithm for the linear SVBP (linear problem in the upper level and a MOLP problem in the lower level) by reformulating the problem as a special bilevel programming problem where the lower level is a parametric linear scalar optimization problem (using the weighted-sum scalarizing approach). The bilevel problem is then transformed into a non-smooth (one level) optimization problem. All the aforementioned approaches deal with the optimistic formulation. 
The pessimistic formulation is more challenging from the theoretical, methodological and computational perspectives. Liu et al. [29] developed necessary optimality conditions for the pessimistic formulation using a scalarization technique (that converts the lower level problem into a parametric program), following an approach related to the one proposed by Dempe et al. [30] for the optimistic formulation. $\mathrm{Lv}$ and Chen [31] proposed a discretization iterative algorithm (inspired by discretization techniques used in semi-infinite programming) to compute the pessimistic optimal solution to a SVBP with a convex lower level problem and no upper level variables in the lower level constraints.

As can be seen by the previous references, theoretical and algorithmic contributions made thus far to solve semivectorial bilevel problems have generally adopted an optimistic approach. The optimistic approach presumes that the follower does not have preferences and the leader is free to select the solution that suits him best among the efficient solutions to the follower. This assumption is, however, unlikely in most practical decision situations. Alves et al. [32] discussed the consequences of assuming an optimistic approach in a real decision situation. The authors also introduced a new solution concept called deceiving solution (the worst outcome of a failed optimistic approach) and developed an algorithm based on particle swarm optimization to approximate the optimistic, pessimistic and deceiving solutions to general semivectorial bilevel problems.

In order to illustrate the limitations of adopting an optimistic formulation and just computing the optimistic solution (optimal solution to the optimistic formulation) as the solution to the SVBP, let us consider the problem under study dealing with the interaction between an electricity retailer and consumers. In this problem, the retailer (upper level decision maker) establishes dynamic electricity prices and wants to maximize profits. The consumer (lower level decision maker) wants to minimize the electricity bill and simultaneously minimize his dissatisfaction associated with the corresponding load scheduling. Suppose that the retailer sets a specific electricity price configuration. For this decision, a set of efficient solutions to the consumer can be computed. At one extreme situation there is the load schedule that minimizes the consumer's electricity cost and, at the other one, there is a load schedule associated with the highest cost but optimal for the comfort objective function. In this problem, higher consumer's cost is in general associated with higher retailer's profit. The optimistic approach assumes that the follower is indifferent between all efficient solutions for that price setting, including those two opposite situations. This leads the retailer to believe that the follower would always disregard cost, just looking at comfort, because this choice would be the best one for the retailer. Actually, accepting an optimistic approach means assuming that the follower is indifferent between optimizing one objective function, optimizing the other objective function or obtaining any compromise between the two objective function values. Therefore, the larger the number of lower level objective functions is, more freedom is given by the optimistic approach to the leader for choosing the solution more convenient to him. In practice this assumption is seldom realistic. Therefore, to provide decision aid to the leader in semivectorial bilevel problems, different types of solutions should be computed, which can give insights to the leader of possible outcomes and ranges of values resulting from different decisions.

The optimistic solution indicates the leader his maximum profit when the follower's decision for each $x$ setting is the best for the leader. Associated with the optimistic approach, the deceiving solution can also be defined, which results whenever the leader makes an optimistic decision and the follower's reaction is against the interests of the leader, i.e., a solution resulting from a failed optimistic approach.

The pessimistic solution is the one that gives the maximum profit for the leader when the follower's decision for each $x$ setting is the worst for the leader. We can further consider the best return of a pessimistic approach, which we call the rewarding solution: this solution is obtained whenever the leader takes a pessimistic approach and the follower's reaction is the most favorable to the leader.

Regarding the formulation (SVBP), where the upper level objective function is to be maximized, these four types of extreme solutions can be defined as:

- the optimistic solution, $\left(x^{o}, y^{o}\right)$, is given by

$$
\max _{x, y}\left\{F(x, y): y \in \Psi_{E f}(x), G(x, y) \leq 0\right\}
$$

- given the optimistic upper level decision $x^{o}$, the deceiving solution is $\left(x^{d}, y^{d}\right)$ where $x^{d}=x^{o}$ and $y^{d}$ is given by $\min _{y}\left\{F\left(x^{o}, y\right): y \in \Psi_{E f}\left(x^{o}\right)\right\}$. 
- the pessimistic solution, $\left(x^{p}, y^{p}\right)$, is given by

$$
\max _{x}\left\{\min _{y}\left\{F(x, y): y \in \Psi_{E f}(x)\right\}: G(x, y) \leq 0\right\}
$$

- given the pessimistic upper level decision $x^{p}$, the rewarding solution is $\left(x^{r}, y^{r}\right)$ where $x^{r}=x^{p}$ and $y^{r}$ is given by $\max _{y}\left\{F\left(x^{p}, y\right): y \in \Psi_{E f}\left(x^{p}\right), G\left(x^{p}, y\right) \leq 0\right\}$.

Note that the deceiving solution may not satisfy the upper level constraints $G(x, y) \leq 0$, i.e. it may be infeasible to the leader, but this information can also be useful to the leader. The value of $F$ in a feasible deceiving solution can be worse (and is never better) than the one in the pessimistic solution. This assertion is justified as follows. Consider $\varphi_{p}(x)=\min _{y}\left\{F(x, y): y \in \Psi_{E f}(x)\right\}$ and let us designate $(x$, $\left.y \in \varphi_{\mathrm{p}}(x)\right)$ as belonging to the pessimistic frontier. The pessimistic solution $\left(x^{p}, y^{p} \in \varphi_{\mathrm{p}}\left(x^{p}\right)\right)$ is the best feasible solution (i.e., it satisfies $G\left(x^{p}, y^{p}\right) \leq 0$ ) according to $F$ on the pessimistic frontier. The deceiving solution $\left(x^{d}, y^{d} \in \varphi_{\mathrm{p}}\left(x^{d}\right)\right)$ also belongs to the pessimistic frontier. Thus, if the deceiving solution is feasible (i.e., it satisfies $G\left(x^{d}, y^{d}\right) \leq 0$ ), it cannot be better than the pessimistic solution, otherwise it would be the pessimistic solution itself. At the best, the deceiving solution is as good as the pessimistic solution.

These solutions are illustrated in Fig. 1, which shows the leader's objective value $(F)$ for sets of efficient solutions to the lower level problem, $\Psi_{E f}(x)$ (vertical line segments), considering different $x$ values. The top dashed curve represents the best solutions for the leader among the efficient solutions to the lower level and the bottom dashed curve represents the corresponding worst solutions. The optimistic solution is the point with highest $F$ on the top curve and the deceiving solution is the point on the bottom curve with the same $x$. The pessimistic solution is the point with highest $F$ on the bottom curve and the rewarding solution is the point on the top curve with the same $x$.

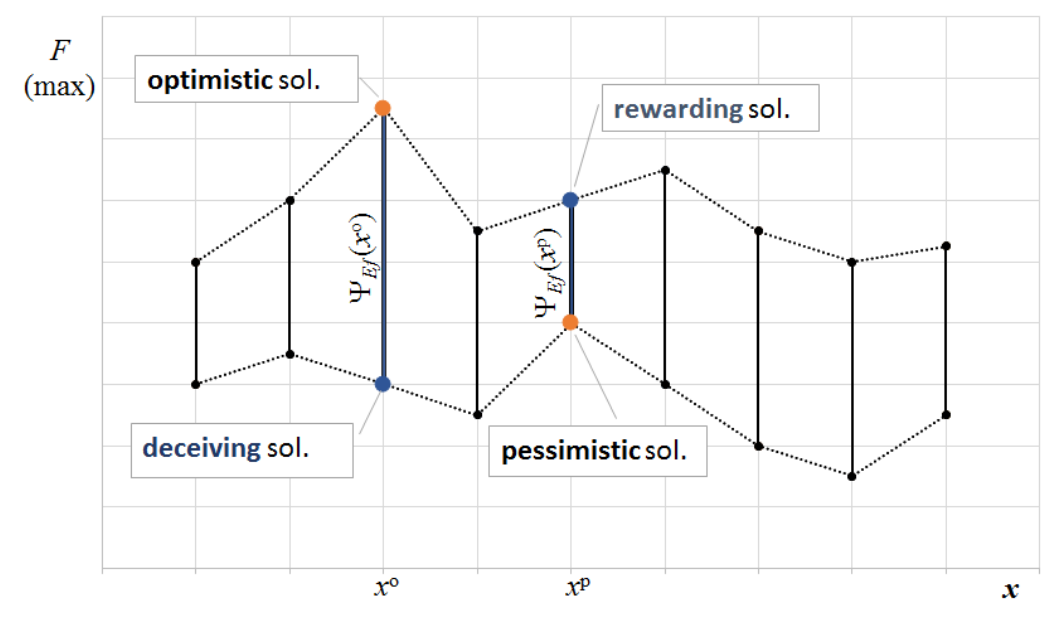

Fig. 1 - Illustration of the optimistic, pessimistic, deceiving and rewarding solutions.

These four types of extreme solutions can be further illustrated using the following semivectorial linear bilevel problem with two lower level objective functions [33]. 


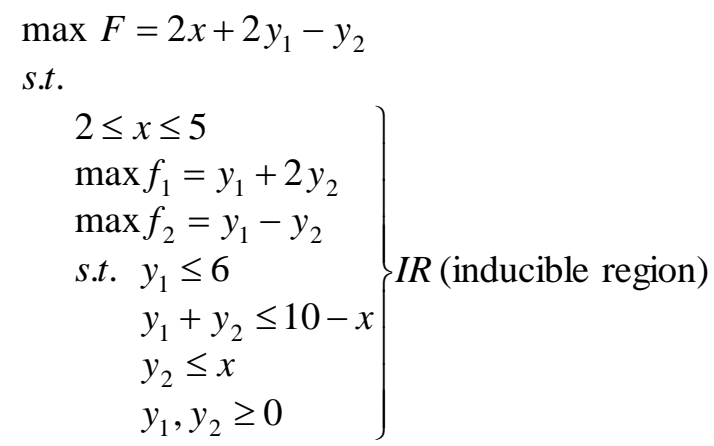

Fig. 2 shows the inducible region $I R$ of the problem in dark gray. The pessimistic solution $\mathrm{P}$ has $\left(x, y_{1}\right.$, $\left.y_{2}\right)=(2,6,2),\left(f_{1}, f_{2}\right)=(10,4), F=14$, and the rewarding solution $\mathrm{R}$ has $\left(x, y_{1}, y_{2}\right)=(2,6,0),\left(f_{1}, f_{2}\right)=(6$, $6), F=16$. This problem has alternative optimistic solutions. All solutions on the edge defined by the vertices $\mathrm{O}$ with $\left(x, y_{1}, y_{2}\right)=(4,6,0)$ and $\mathrm{O}$ ' with $\left(x, y_{1}, y_{2}\right)=(5,5,0)$ give the maximum leader's objective value, $F=20$. If the follower's reaction is not the best to the leader, as expected in the optimistic approach, different worst outcomes for the leader arise for $4 \leq x \leq 5$ thus leading to different deceiving results: from $F(4,6,4)=12$ (in solution D) to $F(5,0,5)=5$ (in solution D'). This illustrative example elucidates the need to explore alternative optimal solutions to the optimistic approach (similar situations may occur for the pessimistic approach). Setting $x=4$ is less risky as it leads to a better deceiving solution, although all values of $x$ on the edge $4 \leq x \leq 5$ lead to the same optimistic objective function value for the leader.

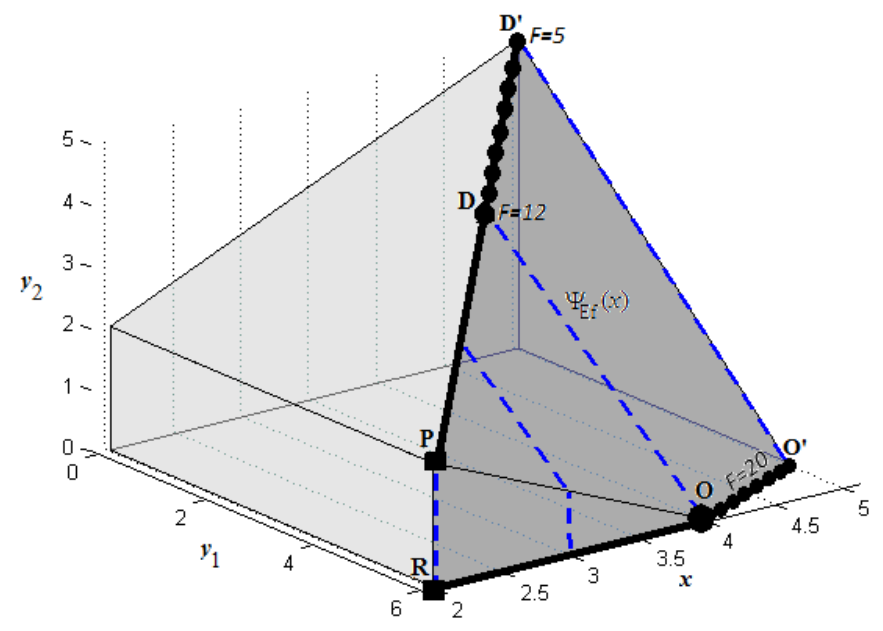

Fig. 2 -Illustrative example of the four extreme solutions with alternative optimistic solutions corresponding to different deceiving outcomes.

\section{A semivectorial bilevel model to optimize electricity dynamic retail pricing}

In this model the retailer's decision consists of determining the optimal price of electricity $x_{i}(€ / \mathrm{kWh})$ in each sub-period $P_{i}(i=1, \ldots, I)$ of the planning period. These prices set by the retailer are bounded by minimum $\left(\underline{x}_{i}\right)$ and maximum $\left(\bar{x}_{i}\right)$ values in each sub-period; in addition, an average price $\left(x^{A V G}\right)$ relative to the entire planning period is imposed. The consideration of maximum, average and minimum prices for the electricity tariffs set by the retailer throughout the planning period act as proxies for competition in retail markets. As referred to in [11], these constraints satisfactorily model possible contracts between retailers and consumers able to reschedule the appliance operation periods in face of dynamic tariffs (and thus willing to engage in new time-differentiated tariff schemes instead of flat rate ones). The retailer may establish these constraints based on estimates of wholesale prices and expected profit margins in a competitive retail market. The lower/upper bound prices, the corresponding sub-periods and the average price offered by each retailer are meaningful information to be compared by consumers when making their contracting decisions. Therefore, the agreement on this set of constraints seems a realistic way to 
model commercial offers in a competitive electricity retail market, while ensuring end-users are not exposed to short-term price volatility of wholesale markets.

The consumer has control over a set of shiftable loads (appliances), $j=1, . ., J$, whose operation cycles can be set within allowable time slots $T_{j}$ according to his preferences and comfort requirements regarding the provision of energy services (hot water, laundry, electric vehicle charge, etc.). A base load corresponding to non-controllable appliances is also considered, which cannot be scheduled by the consumer's energy management system. In face of the electricity prices established by the retailer, the consumer aims to determine the intervals of time each appliance $j$ should operate to minimize the electricity bill and the dissatisfaction associated with not operating it in the preferred comfort intervals within $T_{j}$.

Data:

$T=$ number of intervals (minutes, quarter-hour, half-hour or other period of time) of the planning period $(t=1, \ldots, T)$. Let $\mathrm{T}=\{1, \ldots, T\}$. Let $\hbar$ be the duration of one interval as a fraction of one hour (to accommodate the most convenient discretization of the planning period).

$J=$ number of shiftable loads to be managed by the consumer $(j=1, \ldots, J)$.

$I=$ number of sub-periods of time $P_{i} \subset \mathrm{T}$ in which different prices of electricity (time-of-use tariffs) are charged by the retailer to the consumer $(i=1, \ldots, I)$.

$P 1_{i}, P 2_{i}$ : points in time delimiting each sub-period $P_{i}, i=1, \ldots, I$, such that $P_{i}=\left[P 1_{i}, P 2_{i}\right]$ and $\bigcup_{i=1}^{I} \mathrm{P}_{i}=\mathrm{T}$. Let $\bar{P}_{i}$ denote the amplitude of $P_{i}$, i.e. $\bar{P}_{i}=P 2_{i}-P 1_{i}+1$.

$\underline{x}_{i}=$ minimum price charged to the consumer in sub-period $P_{i}(€ / \mathrm{kW} h)$.

$\bar{x}_{i}=$ maximum price charged to the consumer in sub-period $P_{i}(€ / \mathrm{kW} h)$.

$x^{A V G}=$ average price charged to the consumer in $\mathrm{T}$.

$\pi_{t}=$ price the retailer purchases energy in the spot market at time $t \in \mathrm{T}(€ / \mathrm{kW} h)$.

$C_{t}=$ contracted power by the consumer at time $t \in \mathrm{T}(\mathrm{kW})$.

$b_{t}=$ non-controllable base load at time $t \in \mathrm{T}(\mathrm{kW})$, i.e. amount of load that cannot be scheduled by the consumer's energy management system.

$d_{j}=$ duration of the operation cycle of shiftable load $j(h)$.

$g_{j r}=$ power requested by load $j$ at time $r$ of its operation cycle $\left(r=1, \ldots, d_{j}\right)(\mathrm{kW})$.

$T_{j}=\left[T 1_{j}, T 2_{j}\right] \subseteq \mathrm{T}$ : time slot in which load $j$ is allowed to operate, although there may exist different degrees of satisfaction (or dissatisfaction) to the consumer for the operation of load $j$ within $T_{j}$.

$s_{j t}=$ penalty associated with the operation of load $j$ at time $t \in T_{j}$. This is a degree of dissatisfaction, which varies from 0 to $100 . s_{j t}=0$ corresponds to the time intervals the consumer specifies as most preferred to allocate load $j ; s_{j t}=100$ corresponds to the maximum dissatisfaction, although the consumer still allows load $j$ to operate in these time intervals ( $s_{j t}$ is only defined for $t$ within the time slot $T_{j}$ ).

To avoid ambiguity between points in time of the operation cycle of a given load and points in time of the planning period, we refer to the time $r$ of an operation cycle as "stage $r$ ".

\section{Upper level decision variables:}

$x_{i}=$ price charged by the retailer to the consumer during sub-period $P_{i}(€ / \mathrm{kW} h), i=1, \ldots, I$. 


\section{Lower level decision variables:}

$y_{j r t}=$ binary variable representing whether load $j$ is "off" or "on" at time $t$ of the planning period and stage $r$ of its operation cycle.

In order not to unnecessarily increase the number of $y_{j r t}$ variables, they are defined only for $t$ in the time slot allowed for the operation for each load. Therefore, $y_{j r t}$ are defined for $j=1, \ldots, J, r=1, \ldots, d_{j}, t=$ $\mathrm{T} 1_{j}, \ldots, \mathrm{T} 2_{j}$.

Auxiliary lower level variables:

$p_{j t}=$ power requested to the grid by load $j$ at time $t$ of the planning period $(\mathrm{kW}), j=1, \ldots, J, t=1, \ldots, T$.

It is necessary to define whether the operation cycle of load $j$ is "on" or "off" at time $t$ to define several expressions of the model (such as defining the auxiliary variables $p_{j t}$ and the lower level dissatisfaction objective function). This role can be expressed by $\sum_{r=1}^{d_{j}} y_{j r t}$.

In the semivectorial bilevel model presented below, the objective function at the upper level (1) represents the maximization of the retailer's profit (revenue from selling energy to consumer minus cost of purchasing the energy in the spot market). Constraints (2) to (4) define the upper/lower bounds for the energy prices charged to the consumer in each sub-period $P_{i}$ and set an average price in T.

The objective functions of the lower level problem (consumer's problem) are defined by (5) and (6): the first objective function $f_{1}$ aims to minimize the consumer's electricity cost while the second objective function $f_{2}$ is intended to minimize the consumer's dissatisfaction.

The auxiliary lower level variables $p_{j t}$ are enacted by the lower level variables $y_{j r t}$ through defining constraints (7). Since variables $p_{j t}$ are defined for every $t=1, \ldots, T$, defining constraints comprise two groups: (7) which define $p_{j t}$ for $t$ within the time slot allowed for load $j$ to operate (for which $y_{j r t}$ variables have been defined) and (8) for $t$ outside this time slot for which $p_{j t}$ is always zero.

Constraints (9) impose that the contracted power is not exceeded at any time $t$ of the planning period.

Constraints (10) ensure that, at time $t$, each load $j$ is either "off" or is "on" at only one stage $r$ of its operation cycle.

Constraints (11) ensure that, for each load $j$, if it is "on" at time $t$ and at stage $r \leq d_{j}-1$ of its operation cycle, then it must be also "on" at time $t+1$ and at stage $r+1$.

Constraints (12) ensure each load $j$ is operating at stage $r$ exactly once.

Note that constraints (11) do not prevent that a load $j$ starts at a time after $T 2_{j}-d_{j}+1$ and, as it cannot finish later than $T 2_{j}$, it continues from $T 1_{j}$. For instance, consider that a load $j$ is at stage $r=1$ at $t=\mathrm{T} 22_{j}-1, r=2$ at $t=\mathrm{T} 2_{j}$ and then skips to $r=3$ at $t=1, r=4$ at $t=2$, etc.; this operation scheme is not feasible in practice but it does not violate constraints (11). Thus, constraints (13) are imposed, which ensure that each load $j$ starts its operation (stage $r=1$ ) at most at time $T 2_{j}-d_{j}+1$ so that it can finish until $T 2_{j}$, i.e. within its allowed time slot. Constraints (11) together with (12) and (13) ensure that load $j$ is operating exactly $d_{j}$ consecutive time intervals, forcing $y_{j r t}$ to be 0 when load $j$ is "off".

\section{Semivectorial bilevel model:}

$$
\max _{x} F=\sum_{i=1}^{I} \sum_{t \in \mathrm{P}_{i}} x_{i}\left(b_{t}+\sum_{j=1}^{J} p_{j t}\right)-\sum_{t=1}^{T} \pi_{t}\left(b_{t}+\sum_{j=1}^{J} p_{j t}\right)
$$

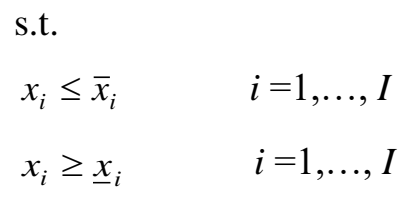




$$
\begin{aligned}
& \frac{1}{T} \sum_{i=1}^{I} \bar{P}_{i} x_{i}=x^{A V G} \\
& \min _{p} f_{1}=\sum_{i=1}^{I} \sum_{t \in \mathrm{P}_{i}} x_{i}\left(b_{t}+\sum_{j=1}^{J} p_{j t}\right) \\
& \min _{y} f_{2}=\sum_{j=1}^{J} \sum_{t=T I_{\mathrm{j}}}^{T 2_{j}}\left(s_{j t} \sum_{r=1}^{d_{j}} y_{j r t}\right)
\end{aligned}
$$

s.t.

$$
\begin{array}{ll}
p_{j t}=\sum_{r=1}^{d j} g_{j r} y_{j r t} & j=1, \ldots, J ; t=T 1_{j}, \ldots, T 2_{j} \\
p_{j t}=0 & j=1, \ldots, J ; t<T 1_{j} \vee t>T 2_{j} \\
\sum_{j=1}^{J} p_{j t}+b_{t} \leq C_{t} & t=1, \ldots, T \\
\sum_{r=1}^{d j} y_{j r t} \leq 1 & j=1, \ldots, J ; t=T 1_{j}, \ldots, T 2_{j} \\
y_{j(r+1)(t+1)} \geq y_{j r t} & j=1, \ldots, J ; r=1, \ldots, d_{j}-1 ; t=T 1_{j}, \ldots, T 2_{j}-1 \\
\sum_{t=T 1_{j}}^{T 2_{j}} y_{j r t}=1 & j=1, \ldots, J ; r=1, \ldots, d_{j} \\
T 2_{j}-d_{j}+1 & \\
\sum_{t=T 1_{j}} y_{j 1 t} \geq 1 & j=1, \ldots, J \\
y_{j r t} \in\{0,1\} & j=1, \ldots, J ; r=1, \ldots, d_{j} ; t=T 1_{j}, \ldots, T 2_{j} \\
p_{j t} \geq 0 & j=1, \ldots, J ; t=1, \ldots, T
\end{array}
$$

\section{A hybrid approach to compute the extreme solutions}

The model (1) - (14) has particularities that can be exploited to design an efficient computational approach to deal with the problem. In what follows, let $y$ denote the lower level vector of variables (composed of the decision variables $y_{j r t}$ and the auxiliary variables $p_{j t}$ ); $x$ is the upper level vector of variables, where $x_{i}, i=1, \ldots, I$ represents the electricity price charged by the retailer to the consumer in each sub-period $\mathrm{P}_{i}$.

The upper level objective function $F$ (retailer's profit) and the first lower level objective function $f_{1}$ (consumer's cost) have a strong correlation, so solutions with higher $F$ values generally present higher $f_{1}$ values. In addition, considering that there is always a time slot larger than or equal to $d_{j}$ for each load $j$ in which the operation of load $j$ totally satisfies the consumer (i.e. $s_{j t}=0$ ), then for each price setting an efficient solution with zero dissatisfaction, $f_{2}=0$, may exist. The minimum value of $f_{2}$ is different from 0 only if all configurations of load operation in slots with total satisfaction violate the contracted power constraint (9). But, even in that case, the minimum value of $f_{2}$ can be known a priori by minimizing $f_{2}$ over (7)-(14) as it does not depend on $x$. Thus, let $\overline{0}$ denote the minimum value of $f_{2}$. This means that, for each $x^{\prime}$, the efficient lower level solutions $y^{\prime}$ that minimize the consumer's dissatisfaction present $f_{2}=\overline{0}$. If there is more than one efficient solution that minimizes $f_{2}$ for a given $x^{\prime}$, all of them surely have the same consumer's cost $f_{1}$ (otherwise they would not be efficient). However, these solutions may present 
different values of the retailer's profit due to the term in (1) corresponding to the purchase of electricity at the spot market.

In the formulations below, $F$ and $f_{1}$ are represented by $F(x, y)$ and $f_{1}(x, y)$ because both depend on $x$ and $y$, but $f_{2}$ only depends on $y$ so it is represented by $f_{2}(y)$.

The optimistic solution to the semivectorial bilevel problem is a solution $\left(x^{o}, y^{o}\right)$ that gives the maximum retailer's profit assuming that the consumer always chooses the efficient solution that most benefits the retailer. This is an efficient solution that minimizes dissatisfaction (i.e., $f_{2}=\overline{0}$ ) or is very close to it. Actually, the second term in (1) may cause that a lower level solution $y^{\prime}$ with maximum cost $f_{1}\left(x^{\prime}, y^{\prime}\right)$ among all $y \in \Psi_{E f}\left(x^{\prime}\right)$ may not be the solution that gives the maximum retailer's profit for that $x^{\prime}$. However, the solution $y \in \Psi_{E f}\left(x^{\prime}\right)$ that gives the maximum $F\left(x^{\prime}, y\right)$ is, in general, among the alternative solutions $\left(x^{\prime}, y\right)$ with $f_{2}=\overline{0}$. Thus, for a given $x^{\prime}$, efficient solutions to the lower level problem that minimize dissatisfaction must be computed if the aim is to determine the optimistic solution. Since the minimum of dissatisfaction is known, these efficient solutions can be obtained by solving problem (P1a), where LLconstraints denotes the set of solutions satisfying the lower level constraints (7) - (14):

$$
\begin{array}{ll}
\min _{y} & f_{1}\left(x^{\prime}, y\right) \\
\text { s.t. } & f_{2}(y)=\overline{0} \\
& y \in \text { LLconstraints }
\end{array}
$$

Problem (P1a) may have alternative optima presenting the same cost and dissatisfaction for the consumer, but presenting different profit for the retailer. A totally optimistic approach (which can be admitted if the leader is somehow able to bias the follower's decision, e.g. by means of side payments) assumes that the consumer will choose the solution with highest profit for the retailer among the tied solutions for the consumer's objective functions. So, in order to approximate the totally optimistic solution, problem (P1b) can be solved instead of (P1a), where $\varepsilon$ is a very small positive constant (e.g., $\left.10^{-4}\right)$. Problem (P1b) was inspired on previous works on bilevel optimization to determine the optimistic solution to bilevel problems with a single objective function at each level [34][35].

$$
\begin{array}{cl}
\min _{y} & f_{1}\left(x^{\prime}, y\right)-\varepsilon F\left(x^{\prime}, y\right) \\
\text { s.t. } & f_{2}(y)=\overline{0} \\
& y \in \text { LLconstraints }
\end{array}
$$

On the other hand, the pessimistic solution to the semivectorial bilevel problem is a solution $\left(x^{p}, y^{p}\right)$ that presents the maximum retailer's profit among the worst solutions (from the retailer's point of view) obtained for each price setting. Thus, it is assumed that the consumer always makes the decision that is worst for the retailer, i.e. he chooses a lower level efficient solution that minimizes cost. For a given price setting $x^{\prime}$, the problem (P2a) can be solved, in which $\rho$ is a small positive weight (e.g., $10^{-2}$ ) assigned to the second objective function to ensure that an efficient solution is obtained to the lower level problem, rather than a weakly efficient solution:

$$
\begin{array}{cl}
\min _{y} & (1-\rho) f_{1}\left(x^{\prime}, y\right)+\rho f_{2}(y) \\
\text { s.t. } & y \in \text { LLconstraints }
\end{array}
$$

Also in this case there may exist alternative efficient solutions that minimize the consumer's cost for price $x^{\prime}$. These solutions correspond to different load schedules with equal comfort and cost for the consumer. If a totally pessimistic approach is adopted (because the leader is risk-averse and/or is not able to influence the choice of the follower), it is assumed that the consumer will choose the solution with minimum $F$ (worst for the retailer) among the alternative optima solutions to (P2a). This solution can be obtained by solving the following problem, where $f_{1}^{a}$ and $f_{2}^{a}$ are the values of $f_{1}$ and $f_{2}$ obtained in (P2a): 


$$
\begin{array}{ll}
\min _{y} & F\left(x^{\prime}, y\right) \\
\text { s.t. } & y \in \text { LLconstraints } \\
& f_{1}\left(x^{\prime}, y\right) \leq f_{1}^{a} \\
& f_{2}(y) \leq f_{2}^{a}
\end{array}
$$

However, since $f_{1}^{a}$ and $f_{2}^{a}$ are floating point numbers, numerical difficulties often exist that may lead to an infeasible problem. Therefore, the resolution of this problem is replaced by the following procedure:

1) After solving (P2a), problem (P2b) is solved with $\varepsilon<\rho\left(\right.$ e.g., $\left.\varepsilon=10^{-4}, \rho=10^{-2}\right)$ :

$$
\begin{array}{ll}
\min _{y} & (1-\rho) f_{1}\left(x^{\prime}, y\right)+\rho f_{2}(y)+\varepsilon F\left(x^{\prime}, y\right) \\
\text { s.t. } & y \in \text { LLconstraints }
\end{array}
$$

Problem (P2b) is a weighted-sum of the lower level objective functions for $\mathrm{x}^{\prime}$, including a very small perturbation associated with the upper level objective function. This perturbation intends to induce a worst solution to the retailer among the alternative optimal solutions to this weighted-sum function.

2) If the optimal solution to (P2b) has $\left(f_{1}, f_{2}\right)=\left(f_{1}^{a}, f_{2}^{a}\right)$ and presents an $F$ value smaller or equal to the $F$ value in the optimal solution to (P2a), then the solution to (P2b) is retrieved. Otherwise, the solution to (P2a) is kept.

A hybrid algorithm has been developed which integrates a genetic algorithm for the upper level search and a MILP solver to optimize the scalarizing problems of the lower level model.

The algorithm presented below is intended to compute solutions that can help the leader (retailer) to delimit the potential outcomes of his decisions. By knowing the optimistic and the deceiving solutions, the retailer acknowledges the result of a completely successful optimistic approach and also the worst possible result if such an approach is followed. By knowing the pessimistic and the rewarding solutions, the retailer perceives the worst and the best outcomes of the most conservative decision. Therefore, these four extreme solutions provide the retailer important insights about the ranges of possible profit values. These are particularly interesting if the leader has no information about the tradeoffs the consumer is willing to make between the electricity bill and the comfort dimension after knowing the electricity prices set by the retailer.

The algorithm described below aims to approximate these four solutions to the semivectorial bilevel problem (1) - (14). Scalarizing problems (P1b) and (P2a,b) are employed for this purpose.

\subsection{Semivectorial Hybrid Genetic Algorithm (SVHGA)}

The genetic algorithm (GA) applies to the upper level problem (1) - (4). Each individual of the population represents an electricity price setting $x^{\prime}=\left(x_{1}^{\prime}, x_{2}^{\prime}, \ldots, x_{I}^{\prime}\right)$. For each $x^{\prime}$ a scalarizing problem concerning the lower level problem (5) - (14) with $x=x^{\prime}$ is exactly solved to obtain an efficient solution $y^{\prime}$ to the lower level problem.

The GA has been coded in Delphi for Windows. The scalarizing lower level problems are MILP problems that are solved by the CPLEX solver, which is called by the GA.

\section{SVHGA:}

Step 1. Create the initial population $P o p$ of $N$ individuals $x^{n}=\left(x_{1}^{n}, x_{2}^{n}, \ldots, x_{I}^{n}\right), n=1, \ldots, N$ with $x_{i}^{n}=$ rand $\left(\underline{x}_{i}, \bar{x}_{i}\right)$; for each $x^{n}$ the Repairing routine (presented below) is applied to ensure that $x^{n}$ also satisfies constraint (4). 
Step 2. For each $x^{n} \in P o p, n=1, \ldots, N$, compute the extreme efficient solutions $\left(x^{n}, y^{n o}\right)$ and $\left(x^{n}, y^{n p}\right)-$ see Fig. 3. These solutions are obtained by solving exactly the problems (P1b) and (P2a,b) for $x=$ $x^{n}$, using the MILP solver.

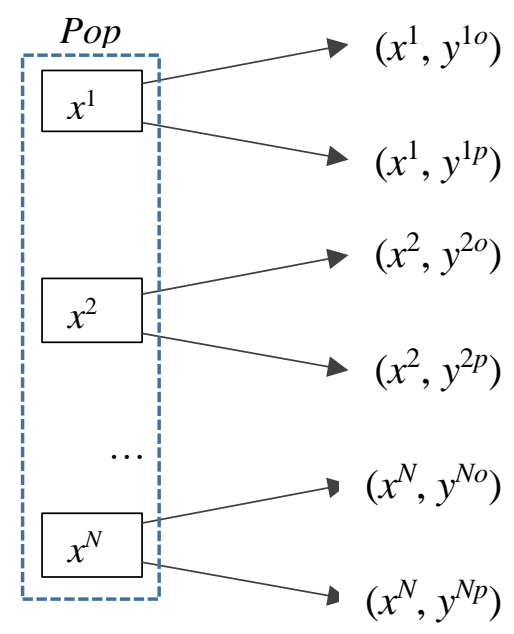

Fig. 3 - Structure of the Population

Step 3. Initialize the incumbent solutions

- Optimistic solution: $\left(x^{o}, y^{o}\right) \leftarrow \arg \max \left\{F\left(x^{n}, y^{n o}\right): x^{n} \in P o p\right\}$

- Pessimistic solution: $\left(x^{p}, y^{p}\right) \leftarrow \arg \max \left\{F\left(x^{n}, y^{n p}\right): x^{n} \in P o p\right\}$

- Deceiving solution: let $k$ be the index of solution $x^{o}$ in Pop such that $x^{k}=x^{o}$; then, $\left(x^{d}, y^{d}\right)=$ $\left(x^{k}, y^{k p}\right)$

- Rewarding solution: let $l$ be the index of solution $x^{p}$ in Pop such that $x^{l}=x^{p}$; then, $\left(x^{r}, y^{r}\right)=$ $\left(x^{l}, y^{l o}\right)$

While the maximum number of iterations is not reached do

Step 4. Create an Offspring population with $N$ children, repeating $N$ times Step 4.1 to Step 4.4:

- 4.1) randomly select one parent $x^{\prime} \in P o p$ and select by binary tournament the other $x^{\prime \prime} \in$ Pop; the tournament is decided by the value of $F\left(x, y^{o}\right)$ in the first $N / 2$ selections and by $F\left(x, y^{p}\right)$ in the remaining $N / 2$ selections. There is equal chance of individuals $x^{\prime}$ or $x^{\prime \prime}$ to be the first or the second parent in the recombination (Step 4.2).

- 4.2) For the selected pair of parents $x^{\prime}$ and $x^{\prime \prime}$, apply one-point crossover to generate a child $x^{c}$; the cutting point is randomly drawn between 1 and $I-1$.

- 4.3) Mutation is then applied to $x^{c}$ with probability $P_{m}$ of changing each gene $i=1, \ldots, I$ of $x^{c}$. For a given $x_{i}^{c}$, mutation consists of adding or subtracting a positive perturbation randomly generated in the range between 0 and $\Delta\left(\bar{x}_{i}-\underline{x}_{i}\right)$ with a parameter $0<\Delta<1$.

- 4.4) Repair $x^{c}$ using the Repairing routine to satisfy constraints (2) - (4) and insert $x^{c}$ in Offspring; if $x^{c}$ is not repairable, generate another $x^{c}$ by repeating the selection of two parents (Step 4.1), recombination (Step 4.2) and mutation (Step 4.3) and the repairing process (Step 4.4).

Step 5. For each $x^{c} \in$ Offspring solve (P1b) and (P2a,b) using the MILP solver to obtain the extreme efficient solutions to the lower level problem: $\left(x^{c}, y^{c o}\right)$ and $\left(x^{c}, y^{c p}\right)$.

Step 6. Update incumbent solutions with Offspring: 
- update the optimistic and deceiving solutions if there is any $\left(x^{c}, y^{c o}\right)$ such that $F\left(x^{c}, y^{c o}\right)>$ $F\left(x^{o}, y^{o}\right)$. If solutions with the same $F$ exist then the one with the best deceiving $F$ is selected.

- update the pessimistic and rewarding solutions if there is any $\left(x^{c}, y^{c p}\right)$ such that $F\left(x^{c}, y^{c p}\right)>$ $F\left(x^{p}, y^{p}\right)$. If solutions with the same $F$ exist then the one with the best rewarding $F$ is selected.

Step 7. Select the individuals of Pop and Offspring to integrate the next population:

Copy to NextPop the $10 \%$ best (w.r.t. $F) x \in P o p \cup$ Offspring among solutions $\left(x, y^{o}\right)$ and the $10 \%$ best (w.r.t. $F) x \in P o p \cup$ Offspring among solutions $\left(x, y^{p}\right)$. The other $80 \%$ elements of NextPop are selected by binary tournaments between one individual of Offspring and one individual of Pop. The result of the tournament is determined by $F\left(x, y^{o}\right)$ in half of the competitions and by $F\left(x, y^{p}\right)$ in the other half. Any individual included in the new population is removed from its original population (Offspring or Pop), so the same individual cannot be selected twice.

$P o p \leftarrow$ NextPop

\section{End While}

Return: $\left(x^{o}, y^{o}\right),\left(x^{p}, y^{p}\right),\left(x^{d}, y^{d}\right),\left(x^{r}, y^{r}\right)$.

Diversity is ensured by the mutation operator and the selection mechanism in Step 7. These diversity enforcing processes are balanced with some elitist pressure in the selection of the population for the next generation.

The Repairing routine (firstly proposed in [12]) works as follows.

Let $x^{\prime}=\left(x_{1}^{\prime}, x_{2}^{\prime}, \ldots, x_{I}^{\prime}\right)$ and let $A$ be the set of indices $i$ of $x_{i}^{\prime}$ that are allowed to be changed. Initially, $A=\{1,2, \ldots, I\}$.

1. For each $i=1, \ldots, I$, if $x_{i}^{\prime}$ is out of the bounds imposed by constraints (2) and (3), then it is pushed to the closest bound $\left(\underline{x}_{i}\right.$ or $\bar{x}_{i}$ ) and $i$ is excluded from the set $A$.

Let $s=\sum_{i=1}^{I} \bar{P}_{i} x_{i}^{\prime}$. If $s=T x^{A V G}$, then stop and return this feasible $x^{\prime}$.

2. If $s \neq T x^{A V G}$, it means that $x^{\prime}$ does not satisfy constraint (4) and the following correction is applied: $x_{i}^{\prime} \leftarrow x_{i}^{\prime}+\frac{T x^{A V G}-s}{\sum_{i \in A} \bar{P}_{i}}$ for all $i \in A$ (if $A=\varnothing$, then stop and discard $x^{\prime}$ as it cannot be repaired).

3. Return to step 1 (since constraints (2) and (3) may no longer be satisfied).

\section{Case study}

The algorithm has been applied to a practical case study whose results are discussed in this section. Data were acquired from actual audit information and some values were estimated. The information to derive the customer's preferences (feasible time slots for load operation) and penalty coefficients to model dissatisfaction with load scheduling has been obtained through audits and surveys in the framework of $\mathrm{PhD}$ theses on Sustainable Energy Systems at the University of Coimbra and reports in the scientific literature [3][36].

\subsection{Problem data}

The input data are similar to the data used in [12] except for the comfort time slots allowed for the operation of each load and the degree of dissatisfaction.

- 24 hours planning period divided into intervals of 15 minutes ( 1 unit of time $t$ ), leading to a planning period of $T=96$ intervals (i.e., units) of time, $\mathrm{T}=\{1, \ldots, 96\}$. Thus, $h=1 / 4 \mathrm{~h}$. 
- 7 sub-periods of time $\mathrm{P}_{i} \subset \mathrm{T}, i=1, . ., 7$, for defining the electricity prices to be charged by the retailer to the consumer.

- The maximum and minimum prices $\left(\bar{x}_{i}\right.$ and $\left.\underline{x}_{i}\right)$ in each sub-period are given in Table A.1 in Appendix. The average price $x^{A V G}$ is $0.116 € / \mathrm{kWh}$.

- The energy prices the retailer has to pay $\left(\pi_{t}\right)$ for the electricity purchased in the spot market are displayed in Table A.2 in Appendix.

- 5 shiftable loads $(J=5)$ : dishwasher, laundry machine, electric water heater $(E W H)$, electric vehicle and clothes dryer. The power required in each stage $r$ of the operation cycle of each load $j$ (i.e. $g_{j r}$ values) is presented in Table A.3 in Appendix.

- The power required by non-controllable base load in each interval $\left(b_{t}, t=1, \ldots, T\right)$ is presented in Table A.4 in Appendix.

- The contracted power $C_{t}$ is $4.6 \mathrm{~kW}$ for $t=28, \ldots, 84$ and $3 \mathrm{~kW}$ for the other $t \in \mathrm{T}$.

- The comfort time slot $\left[T 1_{j}, T 2_{j}\right]$ allowed for each load $j$ and the penalties $\left(s_{j t}\right)$ associated with the operation of each load $j$ at time $t \in T_{j}$ are displayed in Fig. 4. The scale of the $s_{j t}$ values is 0100 , where 0 means total satisfaction and 100 maximum dissatisfaction (minimum comfort) but load $j$ is still allowed to operate at that time $t$. The more or less stringent penalties imposed by these time slots characterize the degree of willingness of consumers to engage in demand response programs regarding each appliance.

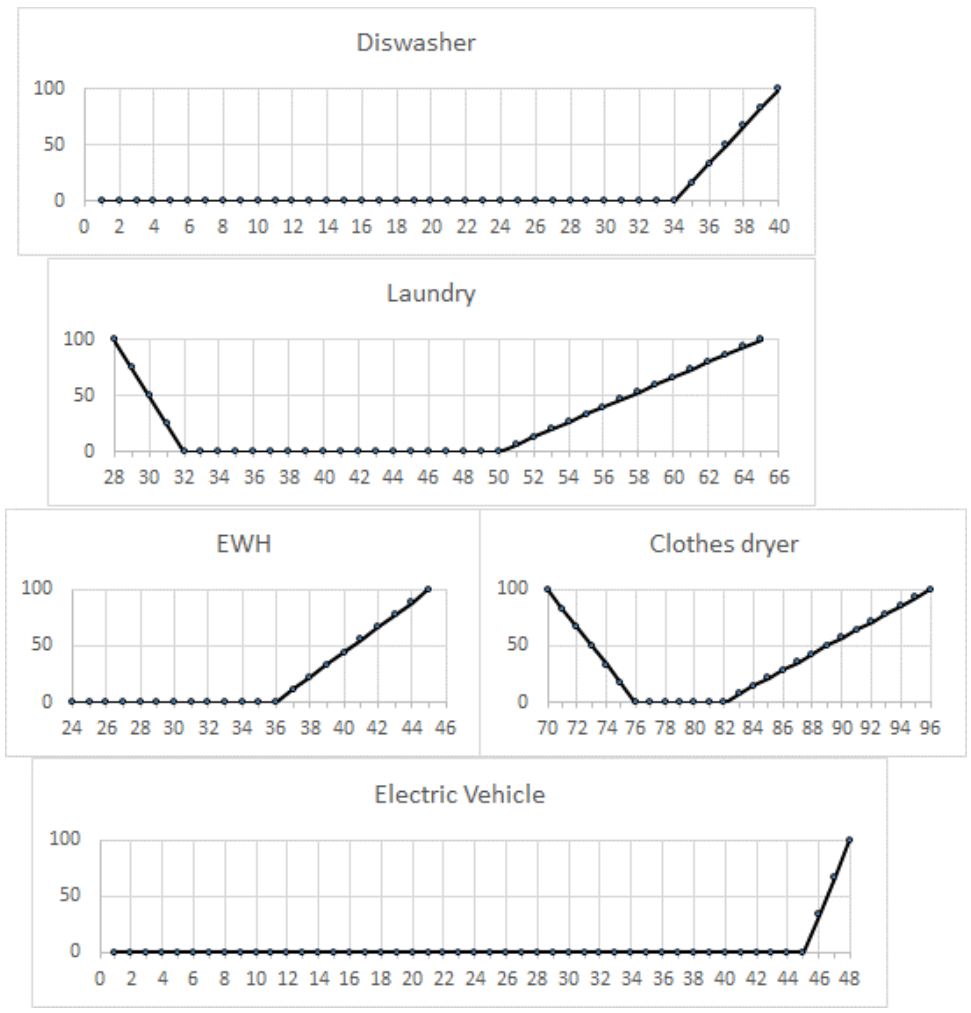

Fig. 4. Comfort time slots allowed for the operation of each load and the degree of dissatisfaction $(0=$ total satisfaction, $100=$ maximum dissatisfaction $)$

\subsection{Parameters}

In the computational simulations of SVHGA, a population size of 30 individuals was considered and 100 iterations of the GA were performed in each run.

In each iteration of SVHGA, three MILP problems are solved by the CPLEX solver for each individual $x$ : (P1b), (P2a) and (P2b). For the data presented in section 5.1, each of these problems has about 2830 variables and 2935 constraints. After the preprocessing phase employed by CPLEX, which eliminates variables and constraints, the adjusted MILP problems to be solved have the following dimensions: 
- (P1b): 1446 variables (1327 binary and 119 continuous) and 1542 linear constraints;

- (P2a), (P2b): 2522 variables (2347 binary and 175 continuous) and 2581 linear constraints.

In order to tune the parameters, extensive experiments were carried out for different values of the mutation probability $\left(P_{m}\right)$ and the $\Delta$ parameter used in the mutation operator (Step 4.3 of the algorithm) considering all combinations of: $P_{m}=0.01, P_{m}=0.05$ and $\Delta=0.1$ to $\Delta=0.7$ with increments of 0.1 (10 independent runs for each combination). Concerning the variation of $P_{m}$, better results were consistently obtained with $P_{m}=0.05$, which is in accordance with the study in [12]. The algorithm seemed not to be very sensitive to the variation of $\Delta$, displaying very small variations for the optimal $F$ values of the optimistic and pessimistic solution (for the best, worst and average values). A slight improvement exists as $\Delta$ increases from 0.1 to 0.4 , but for $\Delta \geq 0.2$ no solution differs from the best optimistic $F$ value obtained in all runs by more than $0.09 \%$. The best and the average values of $F$ of the optimistic solution improve as $\Delta$ increases from 0.1 to 0.4 , but then they seem to worsen slightly for higher values of $\Delta$. The best $F$ of the pessimistic solution is very similar in all combinations of parameters, while the average just improves from $\Delta=0.1$ to $\Delta=0.2$. Therefore, we adopted $P_{m}=0.05$ and $\Delta=0.4$, and results of 20 independent runs are presented henceforth.

We performed 20 independent runs considering two processes of generating the initial population: 1) 30 random feasible individuals; 2) 20 random feasible individuals and 10 individuals "directed" to the upper bound of the price in each sub-period (which requires other prices to be at or close to the lower bound in other sub-periods to ensure feasibility due to the average price constraint). Process 2) introduces a level of greediness in the initial population towards profit maximization (upper level function). The differences between the results of the two processes of generating the initial population are not significant: all lower than $0.03 \%$ for the maximum, minimum, mean and median value of $F$ in the optimistic and the pessimistic solutions. The value of $F$ in the best optimistic solution obtained in the 20 independent runs with the "directed" initial population was slightly better than the best optimistic solution obtained with the pure random initial population; the opposite is true for the pessimistic solution. The results selected for presentation in the paper are the ones obtained with the pure random initial population.

\subsection{Results}

The average computational time for performing one generation with a population of 30 individuals (i.e. Step 4 to Step 7 of the SVHGA, including solving 90 lower level MILP problems) was 23 seconds in a computer Intel Core i7-2600K CPU@3.4GHz, 8 GB RAM with Windows 10. The average time for a complete run (100 generations) was 2295 seconds with a standard deviation of 25.7 seconds. Although the lower level MILP problems have a large number of variables and constraints, in particular binary variables, these problems are solved very fast by CPLEX. Due to the preprocessing phase employed by CPLEX, the optimal solutions to such problems were always found at the root of the branch-and-bound tree in a maximum of 28 iterations of the simplex method.

There is an additional computational effort in solving two problems (P2), i.e., (P2a) and (P2b) for each $x$, but rarely the solution to (P2b) is different from the solution to (P2a). A solution to (P2b) is accepted if it presents equal values of $f_{1}$ and $f_{2}$ with respect to the solution to (P2a) and $F^{2 b} \leq F^{2 a}$. A tolerance of $10^{-4}$ has been admitted to compare the values of $f_{1}$ and $f_{2}$. An accepted solution is considered new if $F^{2 b}<F^{2 a}$ $10^{-5}$. A solution to (P2b) is rejected if $f_{1}$ and $f_{2}$ suffer a variation larger than the tolerance w.r.t. the solution of (P2a). In this experiment with 20 runs, the maximum number of new accepted solutions from (P2b) was 4 in a run with 3030 problems (P2b). The average number of new accepted solutions was 0.8 and the average number of rejected solutions was 55. Total numbers for all computations indicate that $98 \%$ of the solutions obtained with (P2b) are the same as the ones obtained with (P2a) and $99.97 \%$ of the solutions used by the algorithm result from (P2a). Therefore, for the current problem we consider it would be suitable to just solve (P2a) instead of $(\mathrm{P} 2 \mathrm{a})+(\mathrm{P} 2 \mathrm{~b})$ in order to save computational time.

The maximum, minimum, mean and standard deviation of the retailer's profit $(F)$ obtained in the final solutions of the 20 runs are presented in Table 1. This table also shows the results of the particular runs that provided the maximum values, which are marked with ${ }^{(*)}$. The other values in these particular rows that neither are the maxima obtained in the 20 runs nor correspond to the optimistic, deceiving, pessimistic or rewarding final solutions are displayed in grey. The $F$ values are in $€$ and refer to a period 
of 24 hours and a cluster of 1000 consumers with similar consumption and demand response profiles. We observe that the standard deviation is low, i.e. the values of $F$ in the final solutions of these 20 runs are closely around the mean, thus revealing stability of the algorithm.

Table 1 - Values of $F$ obtained in the final solutions of the 20-runs experiment

\begin{tabular}{|c|c|c|c|c|}
\hline \multirow[b]{2}{*}{ Run } & \multicolumn{2}{|c|}{ Optimistic approach } & \multicolumn{2}{|c|}{ Pessimistic approach } \\
\hline & $F$-optimistic & $F$-deceiving & $F$-pessimistic & $F$-rewarding \\
\hline \% & 1922.009 & 1559.066 & 1573.112 & $1909.229^{(*)}$ \\
\hline$\cdots$ & 1919.402 & $1561.991^{(*)}$ & 1574.335 & 1904.282 \\
\hline $\begin{array}{l}\cdots \\
12\end{array}$ & $1923.247^{(*)}$ & 1558.002 & 1573.594 & 1904.429 \\
\hline $\begin{array}{l}15 \\
\ldots\end{array}$ & 1919.626 & 1561.523 & $1574.4355^{(*)}$ & $\underline{1904.204}$ \\
\hline $\operatorname{Maximum}^{(*)}$ & 1923.247 & 1561.991 & 1574.435 & 1909.229 \\
\hline Minimum & 1919.402 & 1557.631 & 1571.905 & 1899.684 \\
\hline Mean & 1921.636 & 1559.220 & 1573.674 & 1903.934 \\
\hline Stand. dev. & 1.088 & 1.158 & 0.562 & 1.717 \\
\hline
\end{tabular}

Care must be taken in reading the "maximum $F$-deceiving" and the "maximum $F$-rewarding" values in Table 1 because they may not correspond to the deceiving and rewarding solutions as defined in Section 2. Although the best $F$-deceiving value found is 1561.991 , this is not obtained for the same $x$ prices as in the optimistic solution. The best solution to the optimistic formulation has an $F$ value of 1923.247 ( $F$ optimistic, which assumes that the consumer schedules his loads in the best way to the leader's profit). Considering the same price setting, the $F$-deceiving is 1558.002 occurring if the consumer schedules his loads in the worst way to the leader. The electricity prices (upper level solution, $x_{i}, i=1, \ldots, 7$ ) for these solutions are presented in the first row of Table 2 .

The best solution to the pessimistic formulation has an $F$ value of 1574.435 ( $F$-pessimistic, the best retailer's profit assuming that the consumer schedules his loads in the worst way to the leader's profit). The corresponding $F$-rewarding is 1904.204 occurring if the consumer, given this price setting, schedules his loads in the best way to the leader. The electricity prices for these solutions are presented in the second row of Table 2 .

As can be seen in Table 2, the electricity prices obtained for the optimistic/deceiving solutions and the pessimist/rewarding solutions only differ in sub-periods $P_{4}, P_{5}$ and $P_{7}$. This similarity can also be observed in the graph of Fig. 5, which depicts the prices in Table 2 and also the minimum and maximum prices allowed in each sub-period of time ( $c f$. Table A.1).

Table 2. Electricity prices $(€ / \mathrm{kWh})$ in the best solution for the optimistic approach and in the best solution for the pessimistic approach

\begin{tabular}{|c|c|c|c|c|c|c|c|}
\hline $\begin{array}{r}\text { Sub-periods } P_{i} \rightarrow \\
{\left[P 1_{i}-P 2_{i}\right]}\end{array}$ & $\begin{array}{c}P_{1} \\
{[1-28]}\end{array}$ & $\begin{array}{c}P_{2} \\
{[29-38]}\end{array}$ & $\begin{array}{c}P_{3} \\
{[39-44]}\end{array}$ & $\begin{array}{c}P_{4} \\
{[45-60]}\end{array}$ & $\begin{array}{c}P_{5} \\
{[61-76]}\end{array}$ & $\begin{array}{c}P_{6} \\
{[77-84]}\end{array}$ & $\begin{array}{c}P_{7} \\
{[85-96]}\end{array}$ \\
\hline \multicolumn{8}{|l|}{ Solution $\downarrow$} \\
\hline Optimistic/Deceiving & 0.1 & 0.24 & 0.12 & 0.120237 & 0.030064 & 0.24 & 0.074266 \\
\hline Pessimistic/Rewarding & 0.1 & 0.24 & 0.12 & 0.100004 & 0.060771 & 0.24 & 0.060300 \\
\hline
\end{tabular}




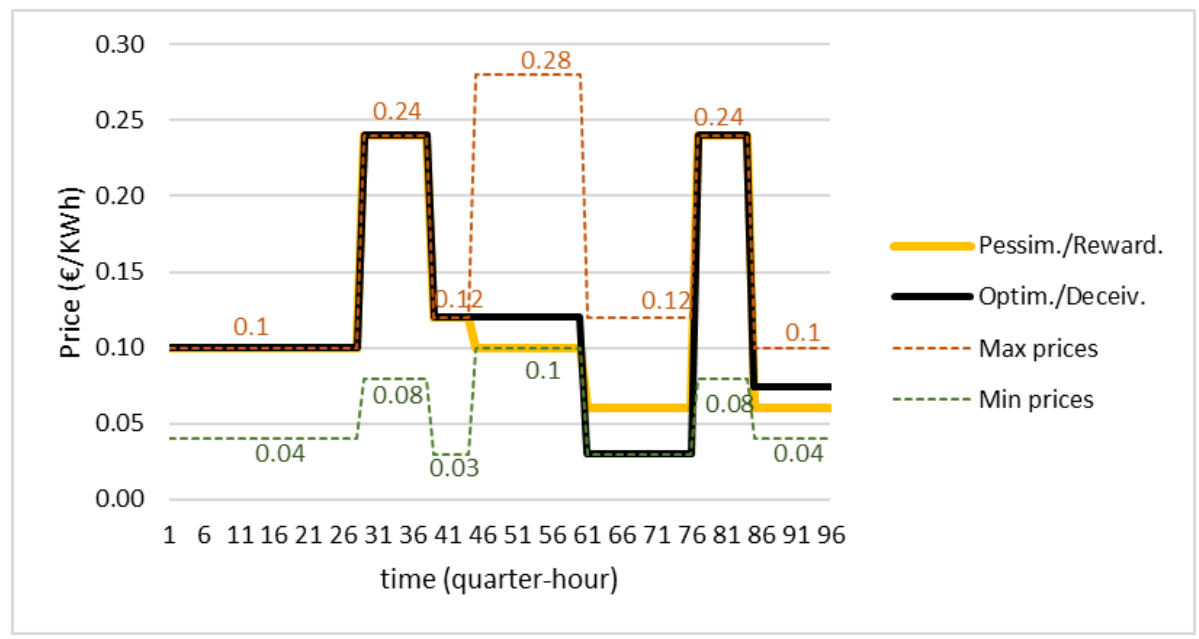

Fig. 5 - Comparison of the electricity prices obtained for the pessimistic and the optimistic approaches.

The objective function values and the load schedules (times for the operation of the loads) in the best optimistic solution obtained in this experiment and the corresponding deceiving solution, as well as the best pessimistic solution and the corresponding rewarding solution, are shown in Table 3.

Table 3. Objective function values and load schedules in the optimistic, deceiving, pessimistic and rewarding solutions.

\begin{tabular}{|c|c|c|c|c|c|c|c|c|}
\hline \multirow[b]{2}{*}{ Solution: } & \multicolumn{3}{|c|}{$\begin{array}{c}\text { Upper level }(F) \text { and lower level }\left(f_{1} \text { and } f_{2}\right) \\
\text { objective function values }\end{array}$} & \multicolumn{5}{|c|}{$\begin{array}{l}\text { [initial time } t_{1} \text { - final time } t_{2} \text { ] } \\
\text { for the operation of each load }\end{array}$} \\
\hline & $\begin{array}{l}\text { Retailer's } \\
\text { profit, } F \\
(€)\end{array}$ & $\begin{array}{l}\text { Consumer's } \\
\text { cost, } f_{1} \\
(€)\end{array}$ & $\begin{array}{l}\text { Consumer's } \\
\text { dissatisfaction, } \\
f_{2}(a)\end{array}$ & Dishwasher & $\begin{array}{l}\text { Laundry } \\
\text { machine }\end{array}$ & EWH & $\begin{array}{l}\text { Electric } \\
\text { vehicle }\end{array}$ & $\begin{array}{r}\text { Clothes } \\
\text { dryer }\end{array}$ \\
\hline Optimistic & 1923.247 & 3434.287 & 0 & [1-5] & [39-44] & [28-32] & [5-40] & [76-78] \\
\hline Deceiving & 1558.002 & 3116.685 & 827.778 & {$[1-5]$} & [60-65] & [39-43] & [5-40] & [74-76] \\
\hline Pessimistic & 1574.435 & 3132.08 & 974.604 & {$[1-5]$} & [60-65] & [41-45] & [5-40] & [85-87] \\
\hline Rewarding & 1904.204 & 3420.186 & 0 & {$[1-5]$} & {$[45-50]$} & [28-32] & [5-40] & [76-78] \\
\hline
\end{tabular}

(a) $0 \equiv$ total consumer's satisfaction, $100 \equiv$ maximum dissatisfaction per interval and per load.

The load diagrams of these solutions are shown in Fig. 6. This figure also displays the dissatisfaction and consumer's electricity cost at each interval of the planning period.
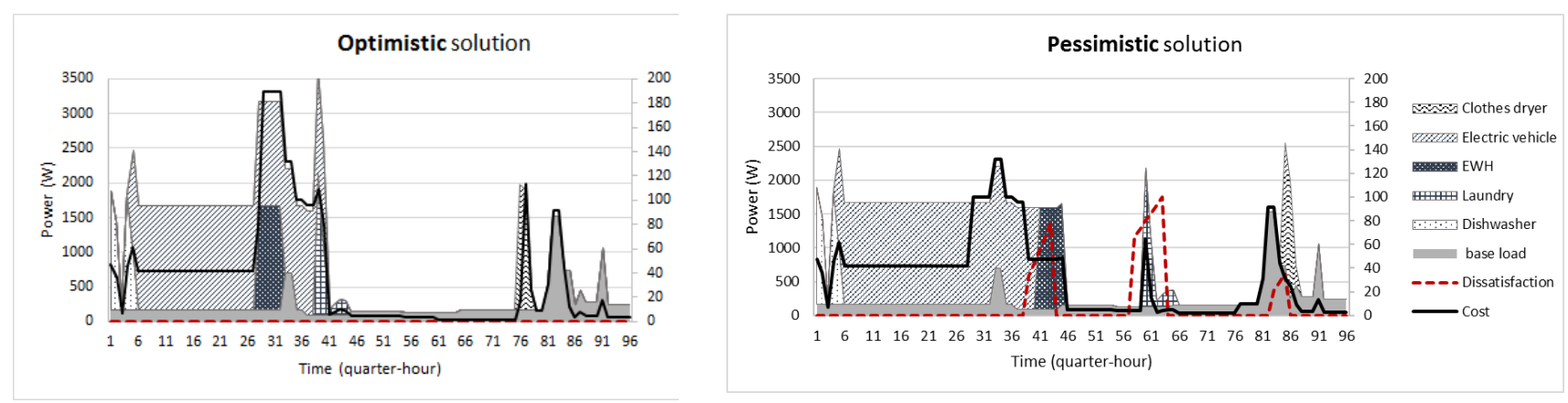

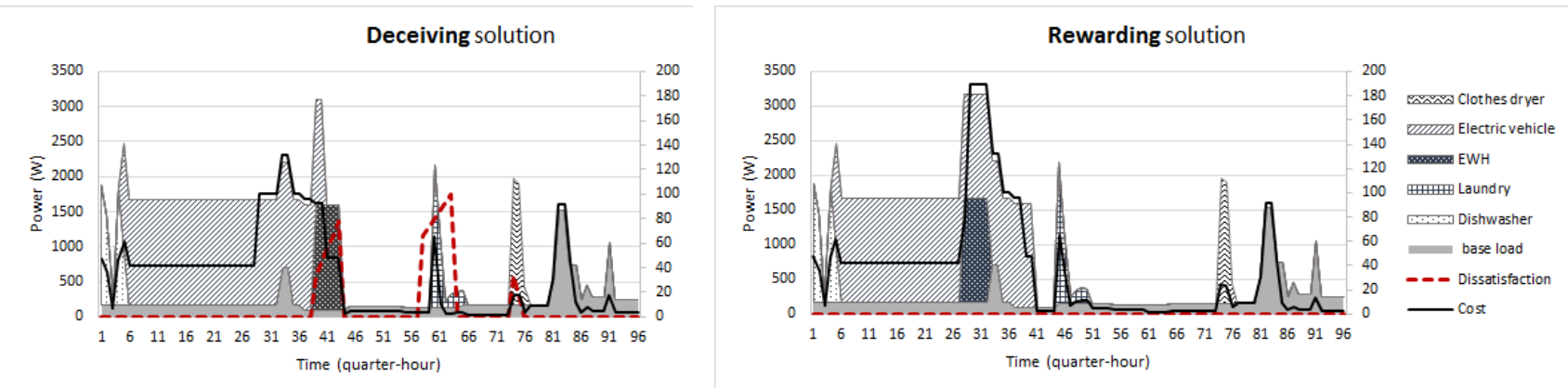

Fig. 6 - Load diagrams of the extreme solutions, showing the consumer's electricity cost and the dissatisfaction at each time.

As Fig. 6 shows, there are close similarities between the optimistic solution and the rewarding solution and between the pessimistic solution and the deceiving solution. These similarities exist regarding the load scheduling patterns and also the time intervals in which the cost and dissatisfaction are more noticeable. The main difference between the load scheduling patterns in the pessimistic and deceiving solutions (the peak in the intervals 39-40 in the deceiving solution) results from the time overlapping of the operation of the EWH and the electric vehicle, which does not exist in the pessimistic solution.

It can be thus observed that there is a low risk of engaging into an optimistic approach and having a deceiving solution with respect to adopting a pessimistic approach. Actually, if the retailer adopts an optimistic perspective and this fails because the consumer schedules his loads in a different way from the optimistic solution, the worst case for the retailer gives a (deceiving) profit of 1558.002. This value is worse but close to the best profit value that the retailer can obtain if he adopts a pessimistic perspective (1574.435).

\subsection{Incorporating consumer's preferences to compute other solutions}

The extreme optimistic, deceiving, pessimistic and rewarding solutions can be regarded as beacon solutions, as they provide the leader information about his profit ranges when adopting two opposed perspectives, optimistic and pessimistic, and the follower's reaction is pro or against the interests of the leader. Other solutions may be analyzed resulting from the incorporation of possible consumer's preferences. The semivectorial bilevel problem is temporarily transformed into a (single-objective) bilevel problem where the lower level problem is a scalarizing problem of the original bi-objective lower level problem. This analysis enables to check how close or far the solutions resulting from the incorporation of different consumer's preferences are from the extreme solutions previously obtained.

The consumer's preferences may be operationalized by means of reference points for the lower level objective function values. These reference points are defined from aspiration levels that the consumer would like to achieve for his electricity bill and the degree of dissatisfaction with the changes of the load operation cycles. The minimization of the augmented Chebyshev distance to the reference point is used to determine, for a given price setting, the consumer's solution closest to those aspiration levels. The bilevel formulation considering the Chebyshev scalarization for the lower level problem is (P3), where $q=\left(q_{1}^{*}, q_{2}^{*}\right)$ denotes the reference point, ULconstraints is the set of solutions satisfying the upper level constraints (2)-(4), $\alpha$ is the variable that gives the Chebyshev distance and $\varepsilon$ is a very small positive constant (e.g., $\left.10^{-4}\right)$. The term $\varepsilon\left(f_{1}(x, y)+f_{2}(y)\right)$ aims at ensuring that an efficient solution is computed rather than a weakly efficient solution only. This term together with $\alpha$ define the augmented Chebyshev distance. 


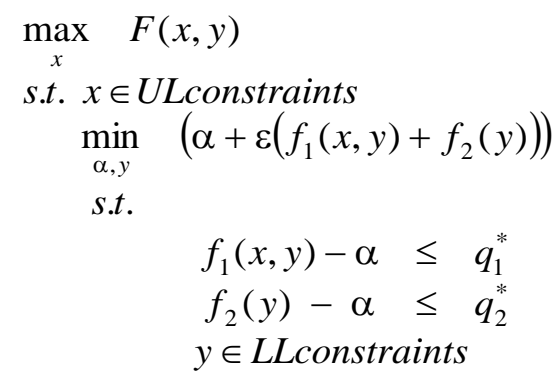

The consumer may also wish to impose bounds on his objective function values. For instance, he would like that his electricity bill was always below a certain level. However, as the electricity prices are controlled by the retailer, there may be circumstances in which those constraints cannot be met. Therefore, we should be careful in adding rigid constraints (as in the $e$-constraint scalarization) into the bilevel formulation, because these constraints may misrepresent the original problem and lead to the computation of a non-optimal solution to the leader. Note that the retailer is the leader and he decides first. He can set electricity prices $x^{\prime}$ for which a given bound for the consumer's cost cannot be met. However, if this bound was incorporated into the model, the algorithm would provide another $x^{\prime \prime}$ (if it exists) as an optimal solution for which the previous bound could be satisfied. This difficulty does not occur if bounds on the dissatisfaction objective function are included because $f_{2}$ is independent of $x$. The inclusion of additional limitations on $f_{2}$ into the Chebyshev scalarizing program (P3) leads to (P4):

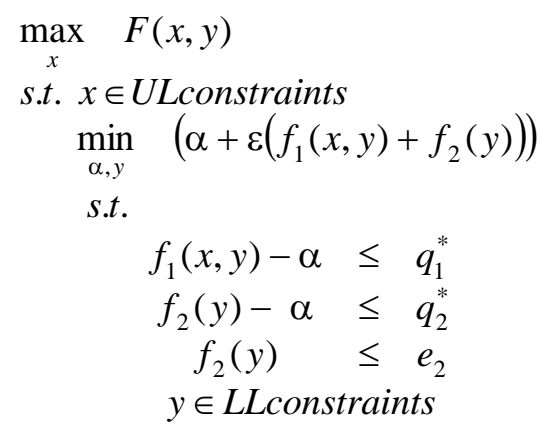

where $e_{2}$ is the upper bound imposed on the consumer's dissatisfaction.

The hybrid GA (HGA) proposed in [12] for single-objective bilevel problems is applied to approximate the optimal solution of each bilevel problem with a scalarizing lower level problem. The best solution (according to the upper level objective function) from 10 independent runs is reported below. In each run 100 iterations of the GA were performed considering a population size of 30 individuals.

\section{- Minimizing the distance to the consumer's 'ideal point'}

The reference point formed by the best values for the consumer obtained in the extreme solutions computed above can be considered as a good representation of the consumer's ideal point: 3116.685 for the cost (minimum cost obtained in the deceiving solution) and 0 for the dissatisfaction (minimum value of dissatisfaction regardless the electricity prices). Thus, problem (P3) is solved with $q^{*}=(3116.685,0)$.

Solution $A\left(F=1777.797, f_{1}=3288.993\right.$ and $\left.f_{2}=85.714\right)$, presented in Tables 5 and 6 , is the best solution according to $F$ obtained in 10 runs of HGA. This solution presents intermediate upper level and lower level objective values w.r.t. to the extreme solutions computed above. However, the consumer may consider this solution a high cost plan. In order to compute other solutions that can reflect the wish of the consumer to reduce cost, the reference level of cost may be decreased with an increase in the reference level of dissatisfaction. In addition, a strict constraint on the dissatisfaction level can be imposed to control the rise of the dissatisfaction objective function. 
- Decreasing the reference level of cost and increasing the reference level of dissatisfaction, imposing an upper bound on dissatisfaction

Considering the reference point $q^{*}=(3000,200)$ and adding $f_{2}(y) \leq 300$, solution $B$ is obtained by solving (P4) using HGA. Solution B, presented in Tables 5 and 6 , is the best solution according to $F$ obtained in 10 runs of the algorithm. As observed in Table 6 , this solution $\left(f_{1}=3170.607\right.$ and $\left.f_{2}=294.445\right)$ cannot attain the objective targets established by the reference point but it also does not take advantage of the upper limit allowed for dissatisfaction (300) to try to reduce the cost.

Therefore, in order to test whether the upper bound $f_{2}(y) \leq 300$ has constrained solution B or not, this constraint is removed and HGA is applied to (P3) with $q^{*}=(3000,200)$, not considering the additional constraint on $f_{2}$.

In this simulation, a solution with a dissatisfaction level higher than 300 is obtained - solution $C$ shown in Tables 5 and 6 . This result enables to conclude that the upper bound of 300 imposed on $f_{2}$ to compute solution B was not redundant, although not binding. Comparing solution $\mathrm{C}$ with solution $\mathrm{B}$, we observe that the consumer's cost has decreased $0.4 \%$ (-13.49 in absolute value) and the consumer's dissatisfaction has increased 11\% (33.33 in absolute value). The load schedule plans are very similar: only the laundry machine starts its operation cycle one interval $(15 \mathrm{~min}$.) later and the clothes dryer starts one interval earlier in solution $\mathrm{C}$ than in solution $\mathrm{B}$. This earlier interval of the clothes dryer operation has some dissatisfaction for the consumer and this is why the dissatisfaction value has increased.

\section{- Minimizing cost imposing an upper bound on dissatisfaction}

If the consumer's preference is to privilege the minimization of cost while bounding the dissatisfaction level, the e-constraint scalarization with the constraint on $f_{2}$ can be used for the lower level problem. This is formulated in problem (P5):

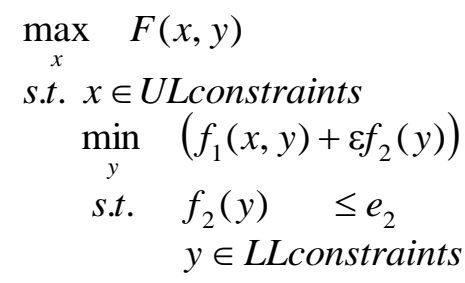

In order to try to further improve consumer's cost with respect to solution C, (P5) is solved with $f_{2}(y) \leq 500$. Again HGA is applied performing 10 independent runs. The best solution obtained to (P5) is solution $D$ presented in Tables 5 and 6. Solution D slightly decreases the consumer's cost with respect to solution $\mathrm{C}(0.04 \% ;-1.28$ in absolute value) and increases the consumer's dissatisfaction in $27.85 \%$ (91.27 in absolute value).

Table 5. Electricity prices $(x)$ in Solutions $A$ to $D$

\begin{tabular}{lccccccc}
\hline $\begin{array}{c}\text { Sub-periods } P_{i} \rightarrow \\
{\left[P 1_{i}-P 2_{i}\right]}\end{array}$ & $\begin{array}{c}P_{1} \\
{[1-28]}\end{array}$ & $\begin{array}{c}P_{2} \\
{[29-38]}\end{array}$ & $\begin{array}{c}P_{3} \\
{[39-44]}\end{array}$ & $\begin{array}{c}P_{4} \\
{[45-60]}\end{array}$ & $\begin{array}{c}P_{5} \\
{[61-76]}\end{array}$ & $\begin{array}{c}P_{6} \\
{[77-84]}\end{array}$ & $\begin{array}{c}P_{7} \\
{[85-96]}\end{array}$ \\
Solution $\downarrow$ \\
\hline Solution A $\left(x^{A}\right)$ & 0.1 & 0.24 & 0.12 & 0.100052 & 0.079724 & 0.231706 & 0.040496 \\
Solution B $\left(x^{B}\right)$ & 0.1 & 0.24 & 0.12 & 0.120004 & 0.033693 & 0.24 & 0.069737 \\
\hline Solution $C\left(x^{C}\right)$ & 0.1 & 0.24 & 0.12 & 0.120059 & 0.055705 & 0.24 & 0.040317 \\
Solution D $\left(x^{D}\right)$ & 0.1 & 0.24 & 0.12 & 0.120144 & 0.049459 & 0.24 & 0.048529 \\
\hline
\end{tabular}


Table 6. Objective function values and load schedules in Solutions $A$ to $D$

\begin{tabular}{l|ccc|ccccc}
\hline & $\begin{array}{c}\text { Retailer's } \\
\text { profit }(F)\end{array}$ & $\begin{array}{c}\text { Consumer's } \\
\text { cost }\left(f_{1}\right)\end{array}$ & $\begin{array}{c}\text { Consumer's } \\
\text { dissatisfaction } \\
\left(f_{2}\right)\end{array}$ & Dishwasher & $\begin{array}{c}\text { Laundry } \\
\text { machine }\end{array}$ & $\begin{array}{r}\text { EWH } \\
\text { Electric } \\
\text { vehicle }\end{array}$ & $\begin{array}{r}\text { Clothes } \\
\text { dryer }\end{array}$ \\
\hline Solution A & 1777.798 & 3288.993 & 85.714 & {$[1-5]$} & {$[45-50]$} & {$[28-32]$} & {$[5-40]$} & {$[85-87]$} \\
Solution B & 1631.357 & 3170.607 & 294.445 & {$[1-5]$} & {$[41-46]$} & {$[39-44]$} & {$[5-40]$} & {$[75-77]$} \\
\hline Solution C & 1617.865 & 3157.115 & 327.778 & {$[1-5]$} & {$[42-47]$} & {$[39-44]$} & {$[5-40]$} & {$[74-76]$} \\
Solution D & 1623.331 & 3155.833 & 419.048 & {$[1-5]$} & {$[39-44]$} & {$[40-45]$} & {$[5-40]$} & {$[85-87]$} \\
\hline
\end{tabular}

As can be observed in Table 5, the electricity prices are particularly close in solutions $\mathrm{C}$ and D. Thus, it can be interesting to further analyze the efficient solutions to the consumer problem that are the best and the worst to the retailer if he adopts such a price pattern. Let us consider the price setting of solution C, i.e., $x^{C}=(0.1,0.24,0.12,0.120059,0.055705,0.24,0.040317)$. Fixing $x=x^{C}$, the best solution $\left(x^{C}, y\right)$ for the retailer is given by $y=\underset{y}{\arg \max }\left\{F\left(x^{C}, y\right): y \in \Psi_{E f}\left(x^{C}\right)\right\}$. We call it a local optimistic solution (which is also a local rewarding solution). Analogously, the worst solution $\left(x^{C}, y\right)$ for the leader is given by $y=\arg \min \left\{F\left(x^{C}, y\right): y \in \Psi_{E f}\left(x^{C}\right)\right\}$. We call it a local pessimistic solution (which is also a local deceiving solution). The values given by these solutions bound the possible outcomes for the retailer from taking the decision of setting prices $x^{C}$. These two local extreme solutions are obtained by solving exactly the MILP problem (P1b) - for obtaining the local optimistic solution - and (P2a) followed by (P2b) - for obtaining the local pessimistic solution - with $x^{\prime}=x^{C}$. The results are shown in Table 7.

Table 7. Objective function values and load schedules in the extreme solutions for $x=x^{C}$

\begin{tabular}{l|ccc|ccccc}
\hline & $\begin{array}{c}\text { Retailer's } \\
\text { profit }(F)\end{array}$ & $\begin{array}{c}\text { Consumer's } \\
\text { cost }\left(f_{1}\right)\end{array}$ & $\begin{array}{c}\text { Consumer's } \\
\text { dissatisfaction } \\
\left(f_{2}\right)\end{array}$ & $\begin{array}{l}\text { Dish- } \\
\text { washer }\end{array}$ & $\begin{array}{c}\text { Laundry } \\
\text { machine }\end{array}$ & $\begin{array}{r}\text { EWH } \\
\text { Electric Clothes } \\
\text { vehicle } \\
\text { dryer }\end{array}$ \\
\hline Local opt./reward. & 1911.739 & 3422.779 & 0 & {$[1-5]$} & {$[39-44]$} & {$[28-32]$} & {$[5-40]$} & {$[76-78]$} \\
Local deceiv./pess. & 1560.567 & 3114.462 & 863.492 & {$[1-5]$} & {$[60-65]$} & {$[39-44]$} & {$[5-40]$} & {$[85-87]$} \\
\hline
\end{tabular}

Remind that the retailer's profit in the (global) optimistic solution is 1923.247 and the retailer's profit in the corresponding deceiving solution is 1558.002 ( $c f$. Table 3). Comparing these values with the ones just obtained, it is realized that the worst values for the retailer's profit are very close, being the local deceiving solution better than the global one in only $0.16 \%$; comparing the global optimistic profit with the local one, the former is better than the latter in about $0.60 \%$. This reinforces the conclusion for the present case study that there is a low risk to the retailer in adopting an optimistic approach and having a deceiving solution with respect to adopting a pessimistic approach or another approach that reflects different possible consumer's preferences. In addition, if the optimistic approach is indeed successful, the retailer can obtain a larger profit than in any other approach.

\section{Conclusions}

Dynamic tariffs are expected to become an applicable pricing scheme in the smart grid context. This type of tariffs provides the price signal incentives for consumers to engage in demand response by means of home energy management systems. In this paper a semivectorial bilevel programming model is proposed to study the interaction between electricity retailers and consumers. Retailers set dynamic tariffs varying in sub-periods during one day aiming to maximize profits. Consumers schedule their loads aiming to minimize the electricity bill and minimize the dissatisfaction in face of his preferences and requirements. 
The lower level (consumer) optimization problem is formulated as a bi-objective MILP problem. A hybrid approach encompassing a genetic algorithm for the upper level (retailer) problem and an exact MILP solver to solve surrogate scalar problems at the lower level is proposed. An illustrative case study is presented and discussed. The extreme optimistic, deceiving, pessimistic and rewarding solutions are computed. In addition, the incorporation of the consumer's preferences is performed to compute other nondominated solutions trading-off the cost and comfort dimensions. This has been accomplished by different means: 1) minimizing the distance to the consumer's "ideal point"; 2) decreasing the reference level of cost and increasing the reference level of dissatisfaction while imposing an upper bound on dissatisfaction; 3) minimizing cost imposing an upper bound on dissatisfaction. The analysis of these results enabled to provide insightful information to assist the retailer decision-making process. In this case study there is a low risk if the retailer embraces an optimistic approach and the consumer reacts in such a way that a deceiving solution is obtained, in comparison with adopting a pessimistic approach. Moreover, if the optimistic approach is successful, according to the consumer's reaction, the retailer can obtain a larger profit than in any other approach.

Further work will assess the performance of other algorithmic approaches to solve the upper level problem and consider clusters of consumers with different energy usage profiles leading to a bilevel multi-follower decision problem.

\section{Acknowledgments}

This work has been partially supported by projects SusCity: Urban data driven models for creative and resourceful urban transitions (MITP-TB/CS/0026/2013), ESGRIDS- Enhancing Smart Grids for Sustainability (SAICTPAC/0004/2015- POCI- 01-0145- FEDER-016434), Suspense - CENTRO-010145-FEDER-000006 and FCT under project grant UID/MULTI/00308/2013.

\section{References}

[1] KU Leuven Energy Institute. Negative electricity market prices. 2014. https://set.kuleuven.be/ei/images/negative-electricity-market-prices.

[2] Utilitywise. UK sees sharp rise in periods of negative pricing during 2016. 2016. https://www.utilitywise.com/2016/06/23/uk-sees-sharp-rise-in-periods-of-negative-pricing-during$2016 /$.

[3] Soares A, Gomes A, Antunes CH, Oliveira C. A Customized Evolutionary Algorithm for MultiObjective Management of Residential Energy Resources. IEEE Trans Ind Informatics 2017;13:492-501. doi:10.1109/TII.2016.2628961.

[4] European Parliamentary Research Service. A New Deal for energy consumers. 2016. http://www.europarl.europa.eu/RegData/etudes/BRIE/2016/573896/EPRS_BRI(2016)573896_EN. pdf.

[5] Zhang G, Zhang G, Gao Y, Lu J. Competitive Strategic Bidding Optimization in Electricity Markets Using Bilevel Programming and Swarm Technique. IEEE Trans Ind Electron 2011;58:2138-46. doi:10.1109/TIE.2010.2055770.

[6] Meng F-L, Zeng X-J. An Optimal Real-time Pricing Algorithm for the Smart Grid: A Bi-level Programming Approach. In: Jones A V, Ng N, editors. 2013 Imp. Coll. Comput. Student Work., vol. 35, Dagstuhl, Germany: Schloss Dagstuhl - Leibniz-Zentrum fuer Informatik; 2013, p. 81-8. doi:http://dx.doi.org/10.4230/OASIcs.ICCSW.2013.81.

[7] Bard JF, Moore JT. A branch and bound algorithm for the bilevel programming problem. SIAM J Sci Stat Comput 1990;11:281-92.

[8] Meng FL, Zeng XJ. An optimal real-time pricing for demand-side management: A Stackelberg game and genetic algorithm approach. 2014 Int. Jt. Conf. Neural Networks, 2014, p. 1703-10. doi:10.1109/IJCNN.2014.6889608.

[9] Meng FL, Zeng XJ. Appliance level demand modeling and pricing optimization for demand response management in smart grid. 2015 Int. Jt. Conf. Neural Networks, 2015, p. 1-8. doi:10.1109/IJCNN.2015.7280432.

[10] Meng FL, Zeng XJ. A bilevel optimization approach to demand response management for the 
smart grid. 2016 IEEE Congr. Evol. Comput., 2016, p. 287-94. doi:10.1109/CEC.2016.7743807.

[11] Zugno M, Morales JM, Pinson P, Madsen H. A bilevel model for electricity retailers' participation in a demand response market environment. Energy Econ 2013;36:182-97. doi:http://dx.doi.org/10.1016/j.eneco.2012.12.010.

[12] Alves MJ, Antunes CH, Carrasqueira P. A hybrid genetic algorithm for the interaction of electricity retailers with demand response. Appl. Evol. Comput. 19th Eur. Conf. (EvoApplications), Lect. Notes Comput. Sci. 9597, Springer; 2016, p. 459-74. doi:10.1007/978-3319-31204-0_30.

[13] Carrasqueira P, Alves MJ, Antunes CH. Bi-level particle swarm optimization and evolutionary algorithm approaches for residential demand response with different user profiles. Inf Sci (Ny) 2017;418-419:405-20. doi:10.1016/j.ins.2017.08.019.

[14] Sekizaki S, Nishizaki I, Hayashida T. Electricity retail market model with flexible price settings and elastic price-based demand responses by consumers in distribution network. Int J Electr Power Energy Syst 2016;81:371-86. doi:http://dx.doi.org/10.1016/j.ijepes.2016.02.029.

[15] Bu S, Yu FR. A Game-Theoretical Scheme in the Smart Grid With Demand-Side Management: Towards a Smart Cyber-Physical Power Infrastructure. IEEE Trans Emerg Top Comput 2013;1:22-32. doi:10.1109/TETC.2013.2273457.

[16] Yang P, Tang G, Nehorai A. A game-theoretic approach for optimal time-of-use electricity pricing. IEEE Trans Power Syst 2013;28:884-92. doi:10.1109/TPWRS.2012.2207134.

[17] Saez-Gallego J, Morales JM, Zugno M, Madsen H. A Data-Driven Bidding Model for a Cluster of Price-Responsive Consumers of Electricity. IEEE Trans Power Syst 2016;31:5001-11. doi:10.1109/TPWRS.2016.2530843.

[18] Dempe S. Bilevel programming: implicit function approach. In: Floudas CA, Pardalos PM, editors. Encycl. Optim., Boston, MA: Springer US; 2009, p. 260-6. doi:10.1007/978-0-38774759-0_44.

[19] Tsoukalas A, Wiesemann W, Rustem B. Global optimisation of pessimistic bi-level problems. Fields Inst Commun 2009;55:1-29.

[20] Wiesemann, W., Tsoukalas, A., Kleniati, P., Rustem B. Pessimistic Bilevel Optimization. SIAM J Optim 2013;23:353-380. doi:10.1137/120864015.

[21] Bonnel H. Optimality conditions for the semivectorial bilevel optimization problem. Pacific J Optim 2006;2:447-68.

[22] Bonnel H, Morgan J. Semivectorial bilevel optimization problem: penalty approach. J Optim Theory Appl 2006;131:365-82. doi:10.1007/s10957-006-9150-4.

[23] Ankhili Z, Mansouri A. An exact penalty on bilevel programs with linear vector optimization lower level. Eur J Oper Res 2009;197:36-41. doi:10.1016/j.ejor.2008.06.026.

[24] Zheng Y, Wan Z. A solution method for semivectorial bilevel programming problem via penalty method. J Appl Math Comput 2011;37:207-19. doi:10.1007/s12190-010-0430-7.

[25] Zheng, Y., Chen, J., Cao X. A Global Solution Method for Semivectorial Bilevel Programming Problem. Filomat 2014;28:1619-27.

[26] Ren A, Wang Y. A novel penalty function method for semivectorial bilevel programming problem. Appl Math Model 2016;40:135-49. doi:10.1016/j.apm.2015.04.041.

[27] Calvete H, Galé C. On linear bilevel problems with multiple objectives at the lower level. Omega 2011;39:33-40. doi:10.1016/j.omega.2010.02.002.

[28] Lv Y, Wan Z. A solution method for the optimistic linear semivectorial bilevel optimization problem. J Inequalities Appl 2014;2014:164. doi:10.1186/1029-242X-2014-164.

[29] Liu B, Wan Z, Chen J, Wang G. Optimality conditions for pessimistic semivectorial bilevel programming problems. J Inequalities Appl 2014;2014:41. doi:10.1186/1029-242X-2014-41.

[30] Dempe S, Gadhi N, Zemkoho A. New optimality conditions for the semivectorial bilevel optimization problem. J Optim Theory Appl 2013;157:54-74. doi:10.1007/s10957-012-0161-z.

[31] Lv Y, Chen J. A Discretization Iteration Approach for Solving a Class of Semivectorial Bilevel Programming Problem. J Nonlinear Sci Appl 2016;9:2888-99.

[32] Alves MJ, Antunes CH, Carrasqueira P. A PSO Approach to Semivectorial Bilevel Programming: Pessimistic, Optimistic and Deceiving Solutions. Proc. Genet. Evol. Comput. Conf. (GECCO 2015), 2015, p. 599-606. doi:10.1145/2739480.2754644.

[33] Alves, M. J., Antunes CH. An illustration of different concepts of solutions in semivectorial bilevel programming. 2016 IEEE Symp. Comput. Intell. (SSCI)., IEEE Xplore; 2016. doi:10.1109/SSCI.2016.7850219. 
[34] Bialas WF, Karwan MH. Two-level linear programming. Manage Sci 1984;30:1004-1020.

[35] Dempe S, Schmidt H. On an algorithm solving two-level programming problems with nonunique lower level solutions. Comput Optim Appl 1996;6:227-49. doi:10.1007/BF00247793.

[36] Soares A, Gomes Á, Antunes CH. Categorization of residential electricity consumption as a basis for the assessment of the impacts of demand response actions. Renew Sustain Energy Rev 2014;30:490-503. doi:https://doi.org/10.1016/j.rser.2013.10.019. 


\section{Appendix}

Table A.1 - Minimum and maximum electricity prices charged to the consumer in each sub-period.

\begin{tabular}{ccccc}
\hline $\begin{array}{c}\text { Sub- } \\
\text { period } P_{i}\end{array}$ & $\begin{array}{r}\text { Start interval, } \\
\mathrm{P} 1_{i} \in\{1, \ldots, 96\}\end{array}$ & $\begin{array}{r}\text { End interval, } \\
\mathrm{P} 2_{i} \in\{1, \ldots, 96\}\end{array}$ & $\begin{array}{c}\text { Minimum price } \\
(€ / \mathrm{kWh})\end{array}$ & $\begin{array}{c}\text { Maximum price } \\
(€ / \mathrm{kWh})\end{array}$ \\
\hline $\mathrm{P}_{1}$ & 1 & 28 & 0.04 & 0.10 \\
$\mathrm{P}_{2}$ & 29 & 38 & 0.08 & 0.24 \\
$\mathrm{P}_{3}$ & 39 & 44 & 0.03 & 0.12 \\
$\mathrm{P}_{4}$ & 45 & 60 & 0.10 & 0.28 \\
$\mathrm{P}_{5}$ & 61 & 76 & 0.03 & 0.12 \\
$\mathrm{P}_{6}$ & 77 & 84 & 0.08 & 0.24 \\
$\mathrm{P}_{7}$ & 85 & 96 & 0.04 & 0.10 \\
\hline
\end{tabular}

Table A.2 - Prices seen by the retailer at the spot market.

\begin{tabular}{cc}
\hline $\begin{array}{c}\text { Start interval }- \text { End interval } \\
(t \in\{1, \ldots, 96\})\end{array}$ & Price $(€ / \mathrm{kWh})$ \\
\hline $1-8$ & 0.05 \\
$9-16$ & 0.035 \\
$17-24$ & 0.045 \\
$25-32$ & 0.065 \\
$33-40$ & 0.075 \\
$41-48$ & 0.08 \\
$49-56$ & 0.09 \\
$57-64$ & 0.1 \\
$65-72$ & 0.11 \\
$73-80$ & 0.085 \\
$81-88$ & 0.08 \\
$89-96$ & 0.1 \\
\hline
\end{tabular}

All prices in Tables A.1 and A.2 are in $€ / \mathrm{kWh}$, so they were then converted to periods of quarter-hour (i.e. divided by 4) to feed the model.

Table A.3 - Operation cycles of the controllable loads.

\begin{tabular}{lc|ccccccc}
\hline & Duration $d_{j}$ & \multicolumn{7}{c}{ Power $g_{j r}(\mathrm{~W})$ in each interval $r=1, \ldots, d_{j}$} \\
Load $j$ & (\# quarter-hours) & $r=1$ & $r=2$ & $r=3$ & $r=4$ & $r=5$ & $r=6$ & $r=7, \ldots, 36$ \\
\hline Dishwasher & 5 & 1724 & 1272 & 104 & 1676 & 799 & & \\
Laundry machine & 6 & 2040 & 1028 & 88 & 180 & 228 & 215 & \\
EWH & 5 & 1500 & 1500 & 1500 & 1500 & 1500 & & \\
Electric vehicle & 36 & 1500 & 1500 & 1500 & 1500 & 1500 & 1500 & 1500 \\
Clothes dryer & 3 & 1808 & 1740 & 282 & & & & \\
\hline
\end{tabular}


Table A.4 - Power requested to the grid by the base (uncontrollable) load.

\begin{tabular}{cc}
\hline $\begin{array}{c}\text { Start interval - End interval } \\
(t \in\{1, \ldots, 96\})\end{array}$ & $\begin{array}{c}\text { Power } b_{t} \\
(\mathrm{~W})\end{array}$ \\
\hline $1-32$ & 166 \\
$33-34$ & 700 \\
$35-36$ & 170 \\
$37-44$ & 92 \\
$45-54$ & 156 \\
$55-64$ & 133 \\
$65-80$ & 159 \\
81 & 522 \\
$82-83$ & 1528 \\
$84-85$ & 742 \\
86 & 249 \\
87 & 452 \\
$88-90$ & 280 \\
91 & 1064 \\
$92-96$ & 241 \\
\hline
\end{tabular}

